\title{
Antipsychotic-Related Stigma and the Impact on Treatment Choices: A Systematic Review and Framework Synthesis
}

\author{
Michael Townsend', Kristin Pareja ${ }^{2}$, Amy Buchanan-Hughes ${ }^{3}$, \\ Emma Worthington ${ }^{4}$, David Pritchett ${ }^{4}$, Malaak Brubaker ${ }^{5}$, Christy Houle ${ }^{6}$, \\ Tenna Natascha Mose ${ }^{6}$, Heidi Waters (iD $^{2}$ \\ 'Gateway Counseling Center, New York, NY, USA; ${ }^{2}$ Department of Global Value and Real World Evidence, Otsuka \\ Pharmaceutical Development \& Commercialization Inc, Princeton, NJ, USA; ${ }^{3}$ Evidence Development Division, Costello \\ Medical Inc, Boston, MA, USA; ${ }^{4}$ Evidence Development Division, Costello Medical Consulting Ltd, Cambridge, UK; \\ ${ }^{5}$ Department of US Medical Affairs, Otsuka Pharmaceutical Development \& Commercialization Inc, Princeton, NJ, USA; \\ ${ }^{6}$ Department of Value and Real World Evidence, Lundbeck Inc, Deerfield, IL, USA
}

Correspondence: Michael Townsend, Email Michael.Townsend@gccnyc.org

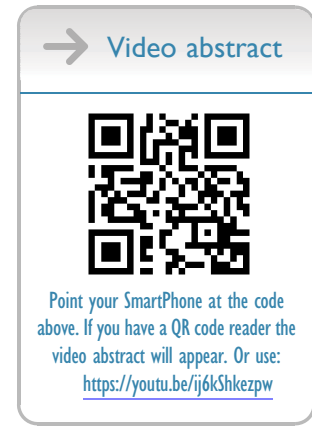

Background: Antipsychotics are a class of medications primarily used to treat individuals with psychotic disorders. They have also been indicated for patients with other psychiatric conditions, such as post-traumatic stress disorder and major depressive disorder. Non-adherence is prominent amongst individuals prescribed antipsychotics, with medication-related self-stigma and social stigma identified as major factors. No previous reviews have focused on stigma associated specifically with antipsychotic medication. This systematic literature review aimed to synthesise evidence on the prevalence of stigmatising attitudes and behaviours related to antipsychotic treatment and understand their impact on antipsychotic treatment initiation and continuation.

Methods: Two independent reviewers screened studies from databases, congress proceedings, ClinicalTrials.gov, and PsychU.org; relevant studies reported quantitative or qualitative data on antipsychotic-related stigma in adults with psychotic disorders, mood disorders, borderline personality disorder or anxiety disorders, or healthcare providers or caregivers of these patients, and any impact on treatment. Framework synthesis facilitated extraction and synthesis of relevant information; quantitative and qualitative data were coded and indexed against a pre-specified thematic framework by two independent reviewers.

Results: Forty-five articles reporting on 40 unique studies were included; 22 reported quantitative data, 16 reported qualitative data, and two reported quantitative and qualitative data relating to antipsychotic-related stigma. Framework synthesis identified four themes: 1) impact of antipsychotic treatment on a) social stigma or b) self-stigma; 2) impact of side effects of antipsychotic treatment on a) social stigma or b) self-stigma; 3) impact of route of administration of antipsychotic treatment on stigma; 4) impact of stigma on the use of antipsychotics.

Conclusion: This systematic literature review found that antipsychotic-related social and self-stigma is a factor in non-adherence to antipsychotics. Further research should examine stigma in a wider range of patients and the extent to which clinicians' treatment decisions are impacted by the potential stigma associated with antipsychotic medications.

Keywords: psychiatric condition, embarrassment, schizophrenia, psychosis, adherence, patient experience

\section{Introduction}

Antipsychotics are a class of medications primarily used to treat psychosis, a mental health condition involving hallucinations, delusions, and disordered speech and thought. They have traditionally been used by patients with schizophrenia, schizoaffective disorder and bipolar disorder. ${ }^{1}$ Beyond their traditional indications, the results of clinical trials have supported antipsychotics as a treatment for individuals with psychiatric conditions outside of psychosis, including those with major depressive disorder (MDD), borderline personality disorder and post-traumatic stress disorder (PTSD). ${ }^{2-6}$ The antidepressant effects of atypical antipsychotics may be attributable to their effects on dopaminergic, serotonergic, glutamatergic, and/or 
gamma aminobutyric acid (GABA)-ergic neurotransmission., ${ }^{2,47}$ Despite their inclusion in major guidelines such as the American Psychiatric Association (APA), National Institute for Health and Care Excellence (NICE) and Canadian Network for Mood and Anxiety Treatments (CANMAT), ${ }^{8-10}$ the use of antipsychotics to treat non-psychotic psychiatric disorders remains controversial, ${ }^{11,12}$ potentially due to contradictory evidence regarding clinical efficacy and the side effects associated with their usage, ${ }^{12-14}$ furthermore, not all of the antipsychotics included in the guidelines mentioned above have US Food and Drug Administration (FDA) approval for the recommended non-psychotic indications.

Non-adherence is a problem in all chronic medical conditions, but it is particularly prominent amongst individuals prescribed antipsychotics and other psychotropic medications. ${ }^{15}$ Existing literature finds that roughly half of patients with psychiatric conditions such as schizophrenia, MDD and bipolar disorder are non-adherent to their medications to treat those disorders. ${ }^{16}$ A comprehensive review which explored longitudinal medication adherence and spanned a range of physical and psychiatric disorders found that, on average, patients took just $58 \%$ of prescribed antipsychotic medication, compared to $65 \%$ of antidepressants, and $76 \%$ of medications to treat physical disorders. ${ }^{17}$ A later systematic literature review (SLR) estimated that adherence in schizophrenia, defined as taking medication at least $75 \%$ of the time, could be as low as $50 \% .{ }^{18}$ Compounded by reduced disease awareness often observed in patients with psychosis, ${ }^{19}$ antipsychotic-related stigma has been identified as a major barrier to treatment adherence in schizophrenia by patients and clinicians, ${ }^{20-22}$ while certain patient studies have reported a significant negative association between stigma and adherence. ${ }^{21,23-26}$ Medication adherence is particularly important for individuals with psychotic disorders such as schizophrenia, as non-adherence may result in symptom recurrence and increase the risk for relapse of psychosis. ${ }^{27,28}$ It is therefore crucial to understand the potential causes of non-adherence in patients with psychiatric disorders, and to develop strategies for encouraging adherence in these patients.

Stigma can be divided into multiple subcategories. ${ }^{29}$ Social stigma refers to negative stereotypes and prejudices held by society that result in discrimination against a specific patient population. This can result in self-stigma, whereby an individual internalises and self-applies these societal views, resulting in low self-esteem, shame and a resigned "why try?" attitude to treatment. Additionally, social stigma can lead to label avoidance, such as an individual rejecting treatment to avoid membership of the stigmatised patient population. To add another layer of complexity, the social stigma associated with psychiatric disorders and their treatment can be exacerbated by undesirable side effects of antipsychotic medication, including weight gain and sexual dysfunction. ${ }^{30,31}$

Numerous reviews have explored the subject of stigma associated with psychiatric disorders, ${ }^{32-36}$ but to the best of our knowledge, no previous SLR has focused on stigma associated with antipsychotic medication. Antipsychotic-related stigma has the potential to influence multiple stages along the treatment journey, including patient willingness to seek treatment, ${ }^{37}$ clinician willingness to recommend and prescribe a specific treatment, patient willingness to accept an antipsychotic prescription, and patient adherence after receiving a prescription. The objective of this SLR was to identify and synthesise qualitative and quantitative evidence on the prevalence of stigmatising attitudes and behaviours related to antipsychotic treatment from a patient, provider and societal perspective, and the impact of such attitudes and behaviours on antipsychotic treatment initiation and continuation. A further objective was to synthesise data from patients with both psychotic and non-psychotic conditions, and to explore any similarities and differences in the experience of antipsychotic-related stigma between these populations.

\section{Methods}

\section{Search Strategy}

The SLR was performed in accordance with a pre-specified protocol and reported in line with the Enhancing Transparency in Reporting the Synthesis of Qualitative Research (ENTREQ) guidelines. ${ }^{38}$ MEDLINE (including MEDLINE In-Process, MEDLINE Daily and MEDLINE Epub Ahead of Print), Embase, PsycINFO, The Cochrane Library (including Cochrane Database of Systematic Reviews [CDSR] and Cochrane Central Register of Controlled Trials [CENTRAL]) and the Database of Abstracts of Reviews of Effects (DARE) were searched on 1st April 2020 (with the exception of PsycINFO, which was searched on 13th May 2020 due to technical issues with the search platform). As the treatment landscape and attitudes to mental illness and treatments have changed over time, the searches were limited to articles published from 2010 onwards. Search terms included combinations of free-text and Medical Subject Heading 
$(\mathrm{MeSH}) /$ Emtree terms related to stigma, relevant psychiatric disorders (eg, anxiety, PTSD, bipolar disorder, MDD, borderline personality disorder, schizophrenia, psychosis) and antipsychotics (Supplementary Tables 1-5). Manual handsearches of various grey literature sources (key congress proceedings from 2017 to March 2020, ClinicalTrials.gov and PsychU.org) were performed (Supplementary Tables 6-8), as well as bibliographies of highly relevant primary studies, systematic reviews and meta-analyses identified during the review.

\section{Study Selection}

Eligibility for inclusion was defined using the SPIDER (Sample, Phenomenon of Interest, Design, Evaluation, Research type) approach. ${ }^{39}$ The sample of interest included: (1) adults with psychotic disorders, mood disorders, borderline personality disorder, or anxiety disorders; or (2) healthcare providers (HCPs) or caregivers of patients with these conditions. Interventional, observational, qualitative, and vignette studies that reported on stigmatising attitudes and behaviours related to antipsychotic treatment (including side effects of antipsychotic treatment) and the impact of this stigma on use of antipsychotics were included in the SLR. Only articles published from 2010 onwards and congress abstracts from 2017 onwards were included. There were no geographical restrictions for included studies; however, only English-language full-texts were included (full eligibility criteria are presented in Supplementary Table 9).

Titles and abstracts of the search results were screened against eligibility criteria by two independent reviewers; discrepancies were resolved by consensus, with arbitration by a third reviewer if necessary. Full-text versions of potentially relevant articles were acquired and screened using the same process.

\section{Thematic Framework}

Framework synthesis is an approach to qualitative data synthesis, developed for applied policy research in the social sciences as a way to facilitate integration of quantitative and qualitative data from diverse sources. ${ }^{40}$ Given the qualitative nature of the questions in this review, framework synthesis was used to facilitate extraction and synthesis of information from the available literature in the most comprehensive and robust way possible. This method of integrating both quantitative and qualitative data into framework synthesis has previously been used in at least two published literature reviews. $^{41,42}$

To facilitate data extraction and synthesis, a preliminary framework of themes that were expected to be identified was determined through a scoping search of the literature and discussion with clinical experts.

\section{Data Extraction, Indexing and Quality Assessment}

The first stage of the data extraction and synthesis process involved the extraction of key information into pre-specified extraction tables in Microsoft Excel. Details on study design, study populations and all relevant quantitative or qualitative results relating to antipsychotic-related stigma were extracted. Examples of relevant quantitative data included the number of patients that have experienced antipsychotic medication-related stigma, or numerical results from questionnaires. Qualitative data mainly comprised of quotations from individual patients describing a specific experience of antipsychotic-related stigma; all relevant quotations from each identified paper were included. Consistent with guidelines from the York University Centre for Reviews and Dissemination, ${ }^{43}$ data extraction in Microsoft Excel was performed by a single individual for each included study, with the extracted information verified by a second independent individual. Discrepancies were resolved by consensus, with arbitration by a third individual if necessary. In cases where multiple publications reported data derived from a single research study, one extraction was performed; data were first extracted from the most detailed publication, and then related publications were checked for additional relevant data. A quality assessment was performed on each included study using the appropriate critical appraisal checklist from the Joanna Briggs Institute. ${ }^{44}$

The thematic framework was developed as a mind-map within Docear, a freely available program that facilitates the thematic mapping of data from multiple articles. ${ }^{45}$ First, the themes from the preliminary framework were included as the backbone of the mind-map. Two reviewers then independently coded and indexed all relevant quantitative or qualitative data from the Excel extraction grid against the pre-specified themes, with data indexed against multiple themes where appropriate. Any new themes or sub-themes that emerged were added to the framework iteratively, and all extracted data 
were considered against both new and pre-specified themes. All members of the project team reviewed the framework during its development and agreed on the final version.

\section{Results}

\section{Included Studies}

Of the 3796 unique records identified through database searches, 836 were selected for full-text review, of which 39 articles met the criteria for inclusion in the review. Six additional articles were identified through hand-searches. Therefore, 45 articles were included, reporting on 40 unique studies (Figure 1).

Twenty-two quantitative studies were included in the SLR, comprised of 16 cross-sectional questionnaire studies, four longitudinal studies, one post-hoc analysis of data from a randomised controlled trial, and one choice-based conjoint analysis. Sixteen cross-sectional qualitative studies used semi-structured interviews or focus groups to explore perceptions and experiences of antipsychotic treatment in smaller samples of patients, psychiatrists or caregivers; in many of these studies, relevant quotations were only reported from one patient. Two studies presented both quantitative and qualitative data relating to antipsychotic-related stigma. Studies were conducted across six continents, with three international studies spanning multiple continents.

Almost all of the included studies focused mainly on patients who had received a diagnosis of psychosis, most often schizophrenia, and were, or had been, treated with antipsychotics. Of the 40 total studies, 30 exclusively included patients with schizophrenia, schizophrenia spectrum disorders, or psychotic disorders, while four studies reported on mixed populations including patients with schizophrenia disorders, bipolar disorder, depression, anxiety, personality disorders, and substance use disorders. Eleven studies additionally or exclusively recruited HCPs, while four studies included caregivers or family members of individuals receiving antipsychotic treatment. No studies reported any direct comparison between patients with psychotic and non-psychotic disorders. Where reported, most recruited patients had been diagnosed with their condition or had received mental health care for several years, ranging from a mean of 5.4 to 21.2 years. The patient sample primarily comprised ( $>60 \%$ ) females in six studies, ${ }^{46-51}$ and males in three studies. ${ }^{52-54}$ In the remaining studies, the gender distribution was relatively equal. Where reported, the mean age of participants ranged from 23 to 50 years. The study design and participant characteristics for all included studies are presented in Table 1.

\section{Thematic Analysis}

The final framework included four overarching themes: (1) impact of antipsychotic treatment on stigma; (2) impact of side effects of antipsychotic treatment on stigma; (3) impact of route of administration of antipsychotic treatment on stigma; and (4) impact of stigma on use of antipsychotics. Themes 1 and 2 were sub-categorised into a) social stigma; and b) self-stigma. The initial framework included Theme 3 as a sub-theme of Theme 1, but the volume of studies identified on this topic suggested that it should be considered separately. Due to the limited number of studies reporting data for patients with non-psychotic conditions, it was not possible to develop any sub-themes comparing psychotic and non-psychotic conditions. Figures 2-9 correspond to each theme/sub-theme identified during thematic analysis. Within these figures, all relevant data, both quantitative and qualitative, are presented for each individual study included in a particular theme (with the corresponding study cited for each data point).

\section{Theme IA: Impact of Antipsychotic Treatment on Social Stigma}

Nine studies reported on the impact of antipsychotic treatment on social stigma: eight qualitative studies and one crosssectional quantitative study. All studies in this theme included patients with psychosis, with only one study also including patients with depression and anxiety. Evidence was mapped to sub-themes including stigmatisation of the treatment name, antipsychotic medications as a symbol of stigmatised psychiatric condition, negative comments relating to regular medication intake, and concerns about being labelled as "crazy" due to antipsychotic treatment (Figure 2).

One study provided quantitative evidence regarding stigmatisation of the treatment name. In this Hong Kong based study, 70 individuals with psychosis were asked whether the label "anti-psyche" drug was bad, to which 52.9\% replied 


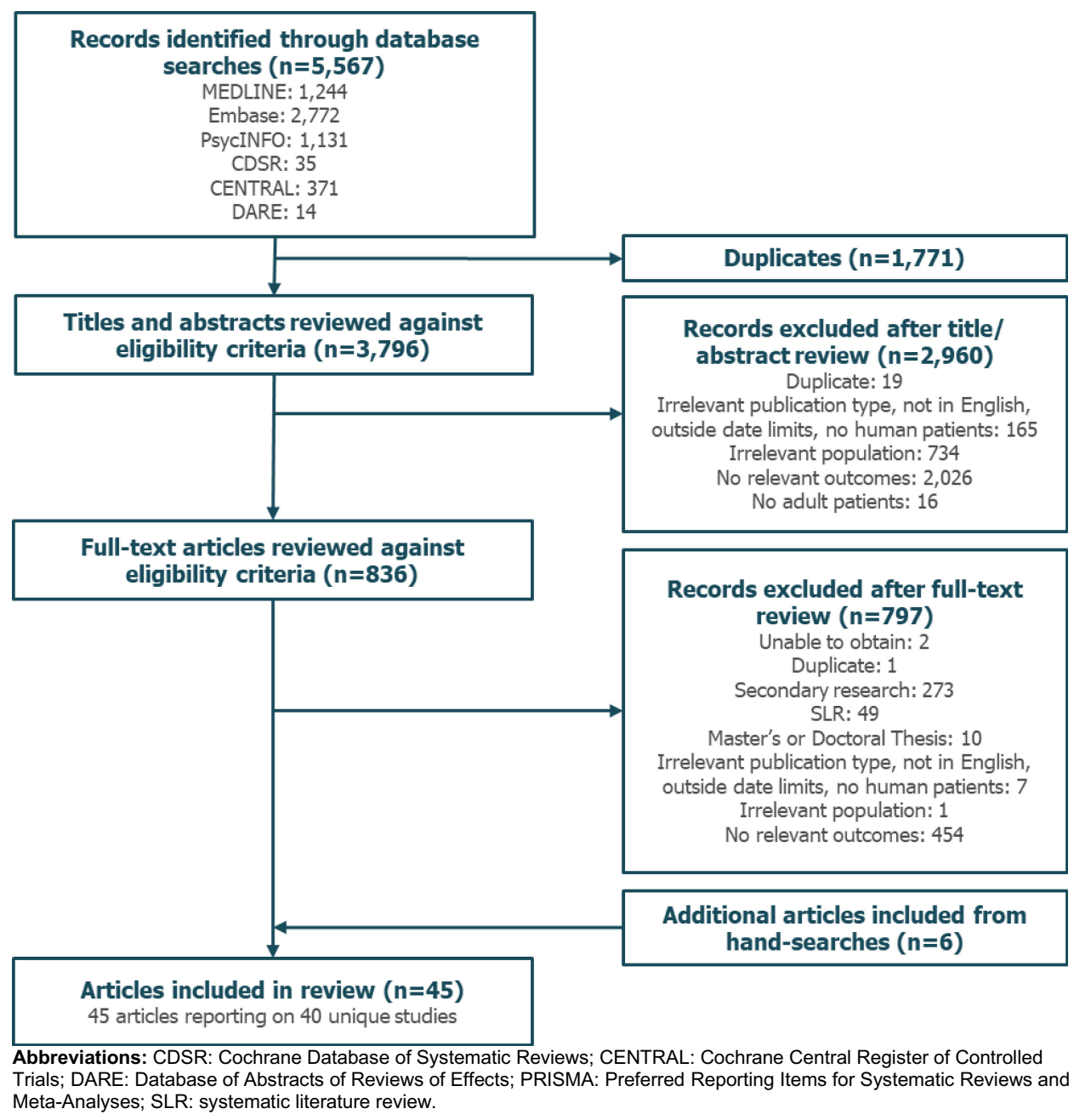

Figure I PRISMA diagram.

that it was. Of that group, $32.9 \%$ said this was due to personal experiences of stigma that this term conveyed to those around them. ${ }^{49}$

Multiple studies reported on discrimination faced by individuals taking antipsychotic medication due to associated stigma. In one study, a participant commented how stigma from others affected their daily life: "[the medication] ... changes other people's attitudes towards me for the worse, makes me feel depressed, sometimes I'm restless sometimes, has a negative effect on my day to day living. ${ }^{55}$,

Similarly, three studies reported on individuals hiding medication consumption for fear of others finding out about their diagnosed psychiatric condition, ${ }^{56-58}$ with one study quoting a clinician as saying "my patient is hiding that he takes medications because he doesn't want to be known to have mental illness. He says that the women will deny him a date". 56

Two additional studies looked at patients' concerns about being labelled "crazy" due to taking antipsychotic medication. ${ }^{59,60}$ One study quoted a patient as saying: "you say you take a medicine for the psychiatric emotional system, there are people who get scared: "he's crazy." 59

\section{Theme IB: Impact of Antipsychotic Treatment on Self-Stigma}

Nine studies reported on the impact of antipsychotic treatment on self-stigma: three quantitative studies, four qualitative studies, and one study from which quantitative and qualitative data were extracted. All studies in this theme included 
Table I Characteristics of Included Studies

\begin{tabular}{|c|c|c|c|c|c|}
\hline Study & Country & $\begin{array}{l}\text { Study } \\
\text { Design }\end{array}$ & Population (N) & Theme(s) & Quality Overview \\
\hline \multicolumn{6}{|l|}{ Quantitative } \\
\hline Blackwood $2019^{70}$ & International & $\begin{array}{l}\text { Post-hoc } \\
\text { analysis }\end{array}$ & $\begin{array}{l}\text { Individuals with } \\
\text { schizophrenia }(N=1429)\end{array}$ & 3 & $\begin{array}{l}\text { - All patients diagnosed according to DSM IV criteria, } \\
\text { and all patients were treated with antipsychotics as part } \\
\text { of the larger study } \\
\text { - A Medication Preference Questionnaire was used, } \\
\text { with the question structure and contents clearly } \\
\text { reported, reducing bias } \\
\text { - Potential confounding variables were considered and } \\
\text { adjusted for using regression analyses } \\
\times \text { Limited recruitment, demographic and diagnostic } \\
\text { information was provided for the participants }\end{array}$ \\
\hline Cahling $2017^{67}$ & Sweden & $\begin{array}{l}\text { Cross- } \\
\text { sectional }\end{array}$ & $\begin{array}{l}\text { Schizophrenia or } \\
\text { schizoaffective disorder; } \\
\text { patients on LAls }(n=63) ; \\
\text { patients on oral medication } \\
(n=101) \text { and mental health } \\
\text { professionals }(n=63)\end{array}$ & 3 & $\begin{array}{l}\text { - Eligibility criteria and study participants described } \\
\text { - The exposure was the idea of being treated with LAI } \\
\text { (for patients on oral treatment) which were stated as } \\
\text { part of the questionnaire } \\
\text { - Appropriate statistical analysis } \\
\times \text { Criteria for patient diagnoses not reported } \\
\times \text { A custom questionnaire designed for the study was } \\
\text { administered via semi-structured interviews for the } \\
\text { outcomes of "stigma" and "embarrassment" on a mm } \\
\text { scale (0-100) }\end{array}$ \\
\hline Emsley $2015^{63}$ & International & $\begin{array}{l}\text { Cross- } \\
\text { sectional }\end{array}$ & $\begin{array}{l}\text { Psychiatric nurses } \\
(\mathrm{N}=4 \mid 20)\end{array}$ & I, 3 & $\begin{array}{l}\text { - Several limitations and confounding variables were } \\
\text { listed } \\
\text { - Sufficient demographic information of nurses } \\
\text { provided, including professional experience level and } \\
\text { health practice setting } \\
\times \text { No steps were taken to mitigate the effects of } \\
\text { confounding variables } \\
\times \text { Custom questionnaire used was not validated or } \\
\text { found to be reliable } \\
\times \text { Recruitment of subjects was not described in detail }\end{array}$ \\
\hline Feldhaus $2018^{77}$ & Germany & $\begin{array}{l}\text { Cross- } \\
\text { sectional }\end{array}$ & $\begin{array}{l}\text { Individuals with } \\
\text { schizophrenia or } \\
\text { schizoaffective disorder } \\
\text { who take antipsychotic } \\
\text { medication }(\mathrm{N}=8 \mathrm{I})\end{array}$ & I, 4 & $\begin{array}{l}\text { - Criteria for recruitment were clearly defined and } \\
\text { sufficient detail was collected on the subjects } \\
\text { - Outcomes were collected using a validated } \\
\text { questionnaire } \\
\text { × Psychiatric conditions were self-reported and not } \\
\text { validated }\end{array}$ \\
\hline Geerts $2013^{76}$ & $\begin{array}{l}\text { Netherlands, } \\
\text { Belgium, } \\
\text { Germany, } \\
\text { Italy, UK, } \\
\text { France, and } \\
\text { "the Nordic } \\
\text { Countries" }\end{array}$ & $\begin{array}{l}\text { Choice-based } \\
\text { conjoint } \\
\text { analysis }\end{array}$ & $\begin{array}{l}\text { Physicians and nurses } \\
\text { treating patients with } \\
\text { schizophrenia }(\mathrm{N}=89 \mathrm{I})\end{array}$ & 3,4 & $\begin{array}{l}\text { - The data collection and analysis methods were } \\
\text { described in detail } \\
\times \text { Recruitment of psychiatrists and eligibility criteria } \\
\text { poorly described } \\
\times \text { A custom questionnaire was used to record } \\
\text { responses, increasing risk of bias (authors raise that } \\
\text { description of attributes may influence respondents' } \\
\text { judgement) } \\
\times \text { It was uncertain how valid and reliable the conjoint } \\
\text { analysis was as it only predicts which treatment options } \\
\text { most closely suit HCPs' preferences, and also assumes } \\
\text { that utilities for individual treatment characteristics are } \\
\text { additive, not permitting any exploration of interaction } \\
\text { effects }\end{array}$ \\
\hline
\end{tabular}

(Continued) 
Table I (Continued).

\begin{tabular}{|c|c|c|c|c|c|}
\hline Study & Country & $\begin{array}{l}\text { Study } \\
\text { Design }\end{array}$ & Population (N) & Theme(s) & Quality Overview \\
\hline Grover $2019^{80}$ & India & $\begin{array}{l}\text { Cross- } \\
\text { sectional }\end{array}$ & Psychiatrists $(N=622)$ & 4 & $\begin{array}{l}\text { - Criteria for recruitment was clearly defined and } \\
\text { sufficient detail was collected on the participants } \\
\times \text { Validity and reliability of data collection methods was } \\
\text { not explored } \\
\times \text { Limitations and potentially confounding variables } \\
\text { were not addressed }\end{array}$ \\
\hline Heres $2012^{46}$ & Germany & $\begin{array}{l}\text { Longitudinal } \\
\text { study }\end{array}$ & $\begin{array}{l}\text { Individuals with } \\
\text { schizophrenia treated with } \\
\text { RLAI }(N=60)\end{array}$ & 3 & $\begin{array}{l}\text { - Eligibility criteria and study participants described } \\
\text { - Appropriate statistical analysis conducted } \\
\times \text { It is not clear if any standard criteria were used for } \\
\text { diagnosis of schizophrenia } \\
\times \text { Potentially confounding variables were mentioned but } \\
\text { not addressed in the analysis }\end{array}$ \\
\hline Ho $2018^{62}$ & China & $\begin{array}{l}\text { Longitudinal } \\
\text { study }\end{array}$ & $\begin{array}{l}\text { Individuals with psychotic } \\
\text { disorder }(N=136)\end{array}$ & 1 & $\begin{array}{l}\text { - Outcomes were measured and analysed in an } \\
\text { appropriate manner } \\
\text { - Clear demographic information was included } \\
\times \text { No steps were taken to mitigate potential biases or } \\
\text { confounding effects }\end{array}$ \\
\hline Hui $2016^{78}$ & China & $\begin{array}{l}\text { Longitudinal } \\
\text { study }\end{array}$ & $\begin{array}{l}\text { Individuals with first } \\
\text { episode psychosis treated } \\
\text { with oral antipsychotics } \\
(\mathrm{N}=3 \mid 3)\end{array}$ & 4 & $\begin{array}{l}\text { - Criteria for recruitment was clearly defined and } \\
\text { sufficient detail was collected on the subjects } \\
\text { - Outcomes were collected using a validated } \\
\text { questionnaire } \\
\times \text { No steps were taken to mitigate potential biases or } \\
\text { confounding factors in this study }\end{array}$ \\
\hline Jaeger $2010^{72}$ & Switzerland & $\begin{array}{l}\text { Cross- } \\
\text { sectional }\end{array}$ & $\begin{array}{l}\text { Individuals with } \\
\text { schizophrenic disorder } \\
\text { treated with antipsychotics } \\
(\mathrm{n}=83) \text {, psychiatrists } \\
(\mathrm{n}=81) \text {, relatives }(\mathrm{n}=91)\end{array}$ & 3 & $\begin{array}{l}\text { - Medication history of depot antipsychotics was } \\
\text { confirmed by checking patients' records } \\
\text { - Validated DAI-10 instrument used, reducing risk of } \\
\text { bias } \\
\text { - Eligibility criteria and characteristics of patients were } \\
\text { described } \\
\times \text { Limited details of the HCPs and relatives provided }\end{array}$ \\
\hline James $2012^{73}$ & Nigeria & $\begin{array}{l}\text { Cross- } \\
\text { sectional }\end{array}$ & $\begin{array}{l}\text { Consultant psychiatrists } \\
\text { and trainees }(\mathrm{N}=128)\end{array}$ & 3 & $\begin{array}{l}\text { - Simple summary statistics were used as appropriate } \\
\text { - Confounding variables were considered } \\
\times \text { A pre-existing questionnaire was used, but it was } \\
\text { designed by one of the authors themselves } \\
\times \text { Limited eligibility criteria and demographic } \\
\text { information about the HCPs were provided }\end{array}$ \\
\hline Jimenez-Trevino $2019^{71}$ & NR & $\begin{array}{l}\text { Cross- } \\
\text { sectional }\end{array}$ & $\begin{array}{l}\text { Individuals with } \\
\text { schizophrenia }(\mathrm{N}=45)\end{array}$ & 3 & $\begin{array}{l}\text { - Study participants described } \\
\text { - Outcomes were measured using validated scales } \\
\text { - Appropriate statistical analysis conducted } \\
\times \text { Antipsychotic treatment was not well-described } \\
\times \text { Inclusion/exclusion criteria were not provided }\end{array}$ \\
\hline Kamaradova $2016^{24}$ & $\begin{array}{l}\text { Czech } \\
\text { Republic }\end{array}$ & $\begin{array}{l}\text { Cross- } \\
\text { sectional }\end{array}$ & $\begin{array}{l}\text { Individuals with stabilised } \\
\text { mental disorder }(\mathrm{N}=332)\end{array}$ & I & $\begin{array}{l}\text { - Eligibility criteria and study participants described } \\
\text { - Antipsychotic treatment well-described } \\
\text { - Outcomes were collected using validated } \\
\text { questionnaires and scales } \\
\text { - Appropriate statistical analysis conducted } \\
\times \text { No information provided about confounding factors }\end{array}$ \\
\hline
\end{tabular}

(Continued) 
Table I (Continued).

\begin{tabular}{|c|c|c|c|c|c|}
\hline Study & Country & $\begin{array}{l}\text { Study } \\
\text { Design }\end{array}$ & Population (N) & Theme(s) & Quality Overview \\
\hline Kamei $2020^{47}$ & Japan & $\begin{array}{l}\text { Longitudinal } \\
\text { study }\end{array}$ & $\begin{array}{l}\text { Individuals with } \\
\text { schizophrenia }(\mathrm{N}=32)\end{array}$ & 3 & $\begin{array}{l}\text { - Eligibility criteria and study participants described } \\
\text { - Antipsychotic treatment well-described } \\
\text { - DSM IV guidelines were used to diagnose } \\
\text { schizophrenia and antipsychotic treatment well- } \\
\text { described } \\
\times \text { No statistical analysis } \\
\times \text { Custom questionnaires were used to determine } \\
\text { patient responses to the shame of gluteal vs deltoid LAI } \\
\text { injections }\end{array}$ \\
\hline $\operatorname{Kim} 2013^{68}$ & South Korea & $\begin{array}{l}\text { Cross- } \\
\text { sectional }\end{array}$ & $\begin{array}{l}\text { Individuals with } \\
\text { schizophrenia or } \\
\text { schizoaffective disorder } \\
(\mathrm{N}=99) \text {, psychiatrists } \\
(\mathrm{N}=173)\end{array}$ & 3,4 & $\begin{array}{l}\text { - Patients were all receiving LAls; psychiatrist } \\
\text { experience level with LAI was described } \\
\text { - Appropriate statistical analysis } \\
\times \text { The outcomes were measured using custom } \\
\text { questionnaires } \\
\times \text { Inclusion criteria for psychiatrists were not well- } \\
\text { defined, particularly regarding psychiatrists who were } \\
\text { invited to complete the survey }\end{array}$ \\
\hline Kulkarni $2015^{85}$ & Australia & $\begin{array}{l}\text { Cross- } \\
\text { sectional }\end{array}$ & Psychiatrists $(\mathrm{N}=406)$ & 4 & $\begin{array}{l}\times \text { Outcomes measured using a custom questionnaire, } \\
\text { with no information provided on the validity or } \\
\text { reliability } \\
\times \text { Criteria for inclusion were poorly defined } \\
\times \text { Subjects and setting were not described in detail }\end{array}$ \\
\hline Lau $2015^{49}$ & China & $\begin{array}{l}\text { Cross- } \\
\text { sectional }\end{array}$ & $\begin{array}{l}\text { Individuals with psychosis } \\
(\mathrm{N}=70)\end{array}$ & 1 & $\begin{array}{l}\text { - Demographic data collection appropriate } \\
\times \text { No information provided about confounding factors } \\
\times \text { Custom questionnaire used without validation }\end{array}$ \\
\hline McEvoy $2019^{66}$ & US & $\begin{array}{l}\text { Cross- } \\
\text { sectional }\end{array}$ & $\begin{array}{l}\text { Individuals with bipolar } \\
\text { disorder, schizophrenia or } \\
\text { major depressive disorder } \\
(\mathrm{N}=4 \mid 6)\end{array}$ & 2 & $\begin{array}{l}\text { - Validated instrument used (SW-ISMI) with simple } \\
\text { summary statistics } \\
\text { - Confounding factors identified and accounted for } \\
\times \text { Unclear criteria used for diagnosis of condition and } \\
\text { antipsychotic use }\end{array}$ \\
\hline Moritz $2013^{50}$ & Germany & $\begin{array}{l}\text { Cross- } \\
\text { sectional }\end{array}$ & $\begin{array}{l}\text { Individuals with } \\
\text { schizophrenia treated with } \\
\text { antipsychotics }(\mathrm{N}=\mid 13)\end{array}$ & 4 & $\begin{array}{l}\text { - Appropriate statistical analysis used } \\
\times \text { Criteria for inclusion were poorly defined } \\
\times \text { Subjects and setting were not described in detail } \\
\times \text { Standard criteria for the measurement of } \\
\text { schizophrenia were not described }\end{array}$ \\
\hline Moritz $2014^{51}$ & Germany & $\begin{array}{l}\text { Cross- } \\
\text { sectional }\end{array}$ & $\begin{array}{l}\text { Individuals taking } \\
\text { antipsychotic medication } \\
(\mathrm{N}=91)\end{array}$ & 4 & $\begin{array}{l}\text { - Appropriate statistical analysis conducted } \\
\times \text { Criteria for inclusion were poorly defined } \\
\times \text { Subjects and setting were not described in detail }\end{array}$ \\
\hline Patel $2010^{74}$ & UK & $\begin{array}{l}\text { Cross- } \\
\text { sectional }\end{array}$ & $\begin{array}{l}\text { Consultant psychiatrists } \\
\text { who prescribed LAls } \\
(\mathrm{N}=102)\end{array}$ & 3,4 & $\begin{array}{l}\text { - Simple summary statistics were used } \\
\times \text { The inclusion and exclusion criteria are not clearly } \\
\text { reported, with limited information about the HCPs } \\
\text { provided } \\
\times \text { Outcomes were measured using a custom } \\
\text { questionnaire created by an author of the study which } \\
\text { could result in bias, although sufficient reliability was } \\
\text { stated }\end{array}$ \\
\hline
\end{tabular}

(Continued) 
Table I (Continued).

\begin{tabular}{|c|c|c|c|c|c|}
\hline Study & Country & $\begin{array}{l}\text { Study } \\
\text { Design }\end{array}$ & Population (N) & Theme(s) & Quality Overview \\
\hline Patel $2020^{75}$ & $\begin{array}{l}\text { Austria, } \\
\text { France, } \\
\text { Germany, } \\
\text { Spain, } \\
\text { Sweden, UK }\end{array}$ & $\begin{array}{l}\text { Cross- } \\
\text { sectional }\end{array}$ & Physicians $(\mathrm{N}=136)$ & 3 & $\begin{array}{l}\text { - Eligibility criteria and study participants described } \\
\text { - Simple summary statistics were used for the } \\
\text { information of interest } \\
\text { - Limitations and potential confounders were } \\
\text { considered } \\
\times \text { Outcomes were measured using a custom } \\
\text { questionnaire } \\
\times \text { Physicians invited to the study are those initiated to } \\
\text { the ALTO study, but qualifications for the ALTO study } \\
\text { are not listed }\end{array}$ \\
\hline \multicolumn{6}{|l|}{ Qualitative } \\
\hline Al-HadiHasan $2017^{65}$ & Jordan & $\begin{array}{l}\text { Face-to-face } \\
\text { semi- } \\
\text { structured } \\
\text { interviews }\end{array}$ & $\begin{array}{l}\text { Individuals diagnosed with } \\
\text { schizophrenia }(n=8) \text { and } \\
\text { their primary caregivers } \\
(\mathrm{n}=9)\end{array}$ & 2 & $\begin{array}{l}\text { - Data collection and analysis appropriate } \\
\text { - Relevant conclusions drawn about educating } \\
\text { caregivers } \\
\times \text { Limited supporting data (two participants; one } \\
\text { example quotation) }\end{array}$ \\
\hline Bjornestad $2017^{79}$ & Norway & $\begin{array}{l}\text { Semi- } \\
\text { structured } \\
\text { interviews }\end{array}$ & $\begin{array}{l}\text { Individuals with first- } \\
\text { episode psychosis enrolled } \\
\text { in two naturalist studies } \\
\text { TIPS-I and TIPS-2 ( } \mathrm{N}=20)\end{array}$ & $\mathrm{I}, 4$ & $\begin{array}{l}\text { - Data collection and analysis appropriate } \\
\text { - Relevant conclusions drawn } \\
\times \text { Unclear whether information from all participants is } \\
\text { adequately represented }\end{array}$ \\
\hline Blixen $2020^{56}$ & Tanzania & $\begin{array}{l}\text { Interviews; } \\
\text { focus groups }\end{array}$ & $\begin{array}{l}\text { Individuals with chronic } \\
\text { psychotic disorders }(\mathrm{N}=15 \text {, } \\
\text { but one relevant } \\
\text { participant) }\end{array}$ & I, 3, 4 & $\begin{array}{l}\text { - Data collection and analysis appropriate } \\
\text { - Relevant conclusions drawn } \\
\text { - Setting and interview guide were sufficiently } \\
\text { described } \\
\times \text { Information on the standing of the researcher(s) was } \\
\text { not provided } \\
\times \text { Only one relevant participant }\end{array}$ \\
\hline Bulow $2016^{37}$ & Sweden & Interviews & $\begin{array}{l}\text { Individuals with psychosis } \\
(\mathrm{N}=19)\end{array}$ & I, 2,4 & $\begin{array}{l}\text { - Data collection and analysis appropriate } \\
\text { - Multiple relevant quotations and author } \\
\text { interpretation } \\
\text { - Relevant conclusions drawn } \\
\times \text { No details about the interviewer(s) provided, or } \\
\text { consideration of possible influence of the researchers } \\
\text { on the conduct or findings of the research }\end{array}$ \\
\hline Chiu $2019^{58}$ & Taiwan & $\begin{array}{l}\text { Semi- } \\
\text { structured } \\
\text { interview }\end{array}$ & $\begin{array}{l}\text { Individuals receiving LAls } \\
(\mathrm{N}=14)\end{array}$ & I, 3 & $\begin{array}{l}\text { - Data collection and analysis appropriate } \\
\text { - Relevant conclusions drawn } \\
\times \text { No details about the interviewer(s) provided, or } \\
\text { consideration of possible influence of the researchers } \\
\text { on the conduct or findings of the research }\end{array}$ \\
\hline Das $2014^{69}$ & UK & $\begin{array}{l}\text { Semi- } \\
\text { structured } \\
\text { interviews }\end{array}$ & $\begin{array}{l}\text { Individuals with psychotic } \\
\text { illness }(N=11)\end{array}$ & 3 & $\begin{array}{l}\text { - Qualitative methodology reported and justified } \\
\text { - Multiple example quotations are provided, including } \\
\text { several that are relevant to the question of stigma } \\
\text { caused by antipsychotics } \\
\times \text { No details about the interviewer(s) provided, or } \\
\text { consideration of possible influence of the researchers } \\
\text { on the conduct or findings of the research }\end{array}$ \\
\hline
\end{tabular}

(Continued) 
Table I (Continued).

\begin{tabular}{|c|c|c|c|c|c|}
\hline Study & Country & $\begin{array}{l}\text { Study } \\
\text { Design }\end{array}$ & Population (N) & Theme(s) & Quality Overview \\
\hline de Jager $2018^{54}$ & $\begin{array}{l}\text { The } \\
\text { Netherlands }\end{array}$ & $\begin{array}{l}\text { Semi- } \\
\text { structured } \\
\text { interviews }\end{array}$ & $\begin{array}{l}\text { Individuals diagnosed with } \\
\text { psychotic disorder }(\mathrm{N}=28)\end{array}$ & 2 & $\begin{array}{l}\text { - Data collection and analysis appropriate } \\
\times \text { Limited supporting data (one quotation on an experience } \\
\text { from one participant) } \\
\times \text { No details about the interviewer(s) provided, or } \\
\text { consideration of possible influence of the researchers on } \\
\text { the conduct or findings of the research } \\
\times \text { No conclusions on medication-related stigma were } \\
\text { drawn }\end{array}$ \\
\hline Kaar $2019^{52}$ & UK & Focus groups & $\begin{array}{l}\text { Individuals with a psychotic } \\
\text { disorder taking } \\
\text { antipsychotics }(\mathrm{N}=23)\end{array}$ & 3 & $\begin{array}{l}\text { - Qualitative methodology reported and justified } \\
\times \text { While multiple example participant quotations are } \\
\text { presented, only one is relevant to the objectives of this } \\
\text { review, with no further discussion of medication-related } \\
\text { stigma by authors } \\
\times \text { No details about the interviewer(s) provided, or } \\
\text { consideration of possible influence of the researchers } \\
\text { on the conduct or findings of the research }\end{array}$ \\
\hline Morant $2018^{55}$ & UK & $\begin{array}{l}\text { Qualitative } \\
\text { semi- } \\
\text { structured } \\
\text { interviews }\end{array}$ & $\begin{array}{l}\text { Individuals with a psychotic } \\
\text { condition ( } \mathrm{N}=20 \text {, but one } \\
\text { relevant participant) }\end{array}$ & I & $\begin{array}{l}\text { - Data collection and analysis appropriate } \\
\text { - Relevant conclusions drawn } \\
\text { - Possible biases of the researcher(s) were explored, } \\
\text { and steps taken to mitigate effects } \\
\times \text { Only one relevant participant }\end{array}$ \\
\hline Morrison $2015^{64}$ & Australia & $\begin{array}{l}\text { Semi- } \\
\text { structured } \\
\text { interview }\end{array}$ & $\begin{array}{l}\text { Individuals who were } \\
\text { prescribed antipsychotics } \\
\text { and experienced side } \\
\text { effects }(\mathrm{N}=10)\end{array}$ & I, 2 & $\begin{array}{l}\text { - Data collection and analysis appropriate } \\
\times \text { No details about the interviewer(s) provided, or } \\
\text { consideration of possible influence of the researchers } \\
\text { on the conduct or findings of the research } \\
\times \text { Limited supporting data: data from only one } \\
\text { participant was relevant to this SLR } \\
\times \text { Minimal relevant conclusions drawn }\end{array}$ \\
\hline Murphy $2015^{60}$ & Canada & $\begin{array}{l}\text { Semi- } \\
\text { structured } \\
\text { interview }\end{array}$ & $\begin{array}{l}\text { Young individuals with } \\
\text { diagnosed psychiatric } \\
\text { condition prescribed } \\
\text { antipsychotic }(\mathrm{N}=18)\end{array}$ & 1 & $\begin{array}{l}\text { - Data collection and analysis appropriate } \\
\text { - Relevant conclusions drawn } \\
\times \text { Only one relevant participant } \\
\times \text { No details about the interviewer(s) provided, or } \\
\text { consideration of possible influence of the researchers } \\
\text { on the conduct or findings of the research }\end{array}$ \\
\hline Noor Us Saba $2019^{86}$ & India & $\begin{array}{l}\text { Interviews; } \\
\text { questionnaires }\end{array}$ & $\begin{array}{l}\text { Individuals with } \\
\text { schizophrenia and acute } \\
\text { psychosis who were } \\
\text { prescribed antipsychotics } \\
(\mathrm{N}=72)\end{array}$ & 1 & $\begin{array}{l}\text { - Data collection and analysis appropriate } \\
\text { - Relevant conclusions drawn } \\
\times \text { Authors used a topic guide to minimise bias in } \\
\text { interviews. However, further details about the } \\
\text { interviewer(s) were not provided } \\
\times \text { In-depth information was not provided about the } \\
\text { philosophical perspective guiding data analysis }\end{array}$ \\
\hline Rankovic $2018^{87}$ & Serbia & $\begin{array}{l}\text { Direct } \\
\text { observation; } \\
\text { analysis of the } \\
\text { history of the } \\
\text { disease; semi- } \\
\text { structured } \\
\text { interview }\end{array}$ & $\begin{array}{l}\text { Individuals with } \\
\text { schizophrenia treated with } \\
\text { injections }(n=5) \text {, doctors } \\
(n=2) \text { and nurses }(n=2) \\
\text { trained at administration of } \\
\text { depot injections }\end{array}$ & 3 & $\begin{array}{l}\text { - Qualitative methodology reported and justified } \\
\text { - Medication-related stigma discussed and emphasised by } \\
\text { authors in the discussion } \\
\times \text { No details about the interviewer(s) provided, or } \\
\text { consideration of possible influence of the researchers on } \\
\text { the conduct or findings of the research } \\
\times \text { While multiple example participant quotations are } \\
\text { presented, only one is relevant to the objectives of this } \\
\text { review }\end{array}$ \\
\hline
\end{tabular}

(Continued) 
Table I (Continued).

\begin{tabular}{|c|c|c|c|c|c|}
\hline Study & Country & $\begin{array}{l}\text { Study } \\
\text { Design }\end{array}$ & Population (N) & Theme(s) & Quality Overview \\
\hline Teferra $2013^{57}$ & Ethiopia & $\begin{array}{l}\text { Focus groups; } \\
\text { interviews }\end{array}$ & $\begin{array}{l}\text { Individuals with } \\
\text { schizophrenia }(n=24) \text {, their } \\
\text { caregivers }(n=19) \text {, research } \\
\text { field workers }(n=7) \text { and } \\
\text { health workers }(n=1) \text { in } \\
\text { a rural setting }\end{array}$ & I, 4 & $\begin{array}{l}\text { - Data collection and analysis appropriate } \\
\text { - Relevant conclusions drawn } \\
\text { - Authors used a topic guide to minimise bias in } \\
\text { interviews. However, further details about the } \\
\text { interviewer(s) were not provided }\end{array}$ \\
\hline Usher $2013^{48}$ & Australia & $\begin{array}{l}\text { Semi- } \\
\text { structured } \\
\text { interviews }\end{array}$ & $\begin{array}{l}\text { Individuals with } \\
\text { schizophrenia who gained } \\
\text { weight as a result of taking } \\
\text { antipsychotics }(\mathrm{N}=8)\end{array}$ & 2 & $\begin{array}{l}\text { - Data collection and analysis appropriate } \\
\text { - Multiple relevant quotations and author } \\
\text { interpretation } \\
\text { - Relevant conclusions drawn } \\
\times \text { No details about the interviewer(s) provided, or } \\
\text { consideration of possible influence of the researchers } \\
\text { on the conduct or findings of the research }\end{array}$ \\
\hline Vedana $2013^{59}$ & Brazil & Interviews & $\begin{array}{l}\text { Individuals with } \\
\text { schizophrenia treated with } \\
\text { psychotropic medications, } \\
\text { family members ( } N=36 \text {, but } \\
\text { one relevant participant) }\end{array}$ & $\mathrm{I}, 4$ & $\begin{array}{l}\text { - Data collection and analysis appropriate } \\
\text { - Relevant conclusions drawn } \\
\times \text { No details about the interviewer(s) provided, or } \\
\text { consideration of possible influence of the researchers } \\
\text { on the conduct or findings of the research }\end{array}$ \\
\hline \multicolumn{6}{|c|}{ Quantitative/Qualitative } \\
\hline Weiss $2019^{61}$ & $\begin{array}{l}\text { US, Canada, } \\
\text { Australia, } \\
\text { Denmark, } \\
\text { Norway, Italy, } \\
\text { Spain }\end{array}$ & $\begin{array}{l}\text { Cross- } \\
\text { sectional }\end{array}$ & $\begin{array}{l}\text { Individuals with } \\
\text { schizophrenia treated with } \\
\text { antipsychotics }(\mathrm{N}=435)\end{array}$ & 2 & $\begin{array}{l}\text { - VAS scale of impact of side effects on functioning, } \\
\text { with simple summary statistics } \\
\times \text { Both diagnoses and antipsychotic use was self- } \\
\text { reported through the online survey, so could not be } \\
\text { verified } \\
\times \text { Potential confounding factors were not identified or } \\
\text { adjusted for }\end{array}$ \\
\hline Tranulis 201 I 53 & US & $\begin{array}{l}\text { Semi- } \\
\text { structured } \\
\text { interview }\end{array}$ & $\begin{array}{l}\text { Clinically stable individuals } \\
\text { who were taking } \\
\text { antipsychotic medication } \\
(\mathrm{N}=20)\end{array}$ & I, 3,4 & $\begin{array}{l}\text { - Data collection and analysis appropriate } \\
\text { - Relevant conclusions drawn } \\
\times \text { No details about the interviewer(s) provided, or } \\
\text { consideration of possible influence of the researchers } \\
\text { on the conduct or findings of the research }\end{array}$ \\
\hline
\end{tabular}

Notes: For quality overview, bullet points represent strengths; "x"s represent limitations. Themes: (I) Impact of antipsychotic treatment on stigma; (2) Impact of side effects of antipsychotic treatment on stigma; (3) Impact of route of administration of antipsychotic treatment on stigma; (4) Impact of stigma on use of antipsychotics.

Abbreviations: DSM, Diagnostic and Statistical Manual of Mental Disorders; LAI, long-acting injectable; HCP, healthcare provider; RLAI, risperidone long-acting injectable; DAI, Drug Attitude Inventory; SW-ISMI, Social Withdrawal subscale of the Internalized Stigma of Mental Illness scale; VAS, Visual Analogue Scale; NR: not reported.

patients with psychosis; one study also included patients with conditions such as depression and anxiety, while another study additionally included patients with a diagnosis of bipolar disorder. Evidence was mapped to sub-themes including reduced self-esteem due to the need for antipsychotics, self-stigma due to the perception that taking medication is symbolic of the diagnosed psychiatric condition, and patients labelling themselves as "crazy" due to taking antipsychotics, with another sub-theme looking at some patients' acceptance of their illness and need for medication (Figure 3).

Multiple studies found evidence of a link between the use of antipsychotics and patients' reduced self-esteem, with one US study finding that $35 \%$ of participants taking antipsychotics linked the medications to reduced self-esteem and stigma. ${ }^{61}$ However, another study in China did not find a statistically significant correlation between the use of second-generation antipsychotics and self-stigma in individuals with psychotic disorders $(\mathrm{p}=0.610){ }^{62}$ A different study found that the most important predictor of the total Internalised Stigma of Mental Illness (ISMI) inventory score was the use of antipsychotics $(\mathrm{p}=0.007) .{ }^{24}$

When psychiatric nurses in 29 countries were asked "what percentage of the patients with schizophrenia you have seen in the past month has at any time felt embarrassed or upset at having to take tablets every day for their illness?" 

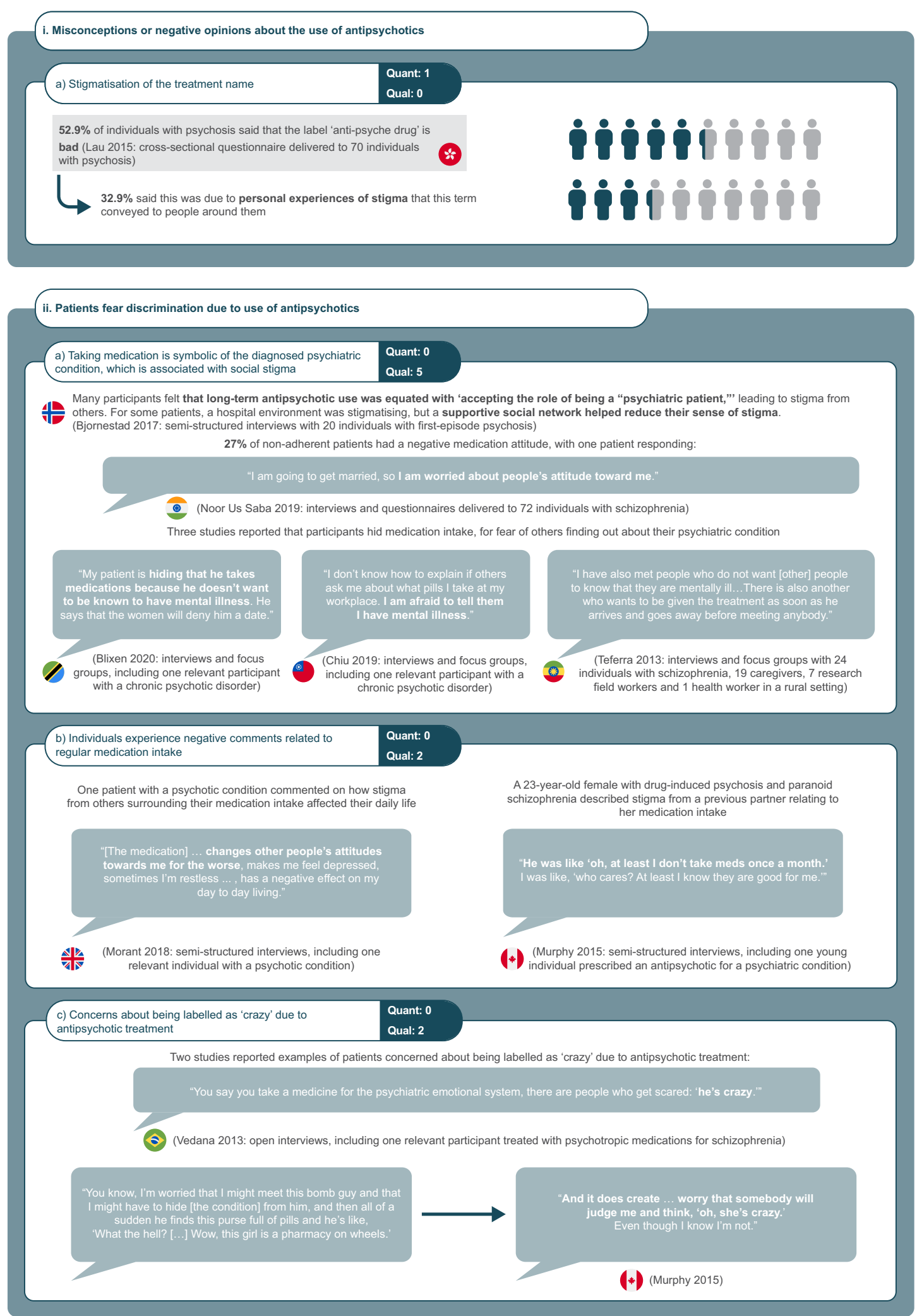

Figure 2 Qualitative and quantitative data for Theme IA: Impact of antipsychotic treatment on social stigma. All qualitative and quantitative data relating to the impact of antipsychotic treatment on social stigma are presented. Quotations from qualitative studies as well as quantitative results are recorded along with the study design, population, and location (where applicable; represented by country flags) for each. 


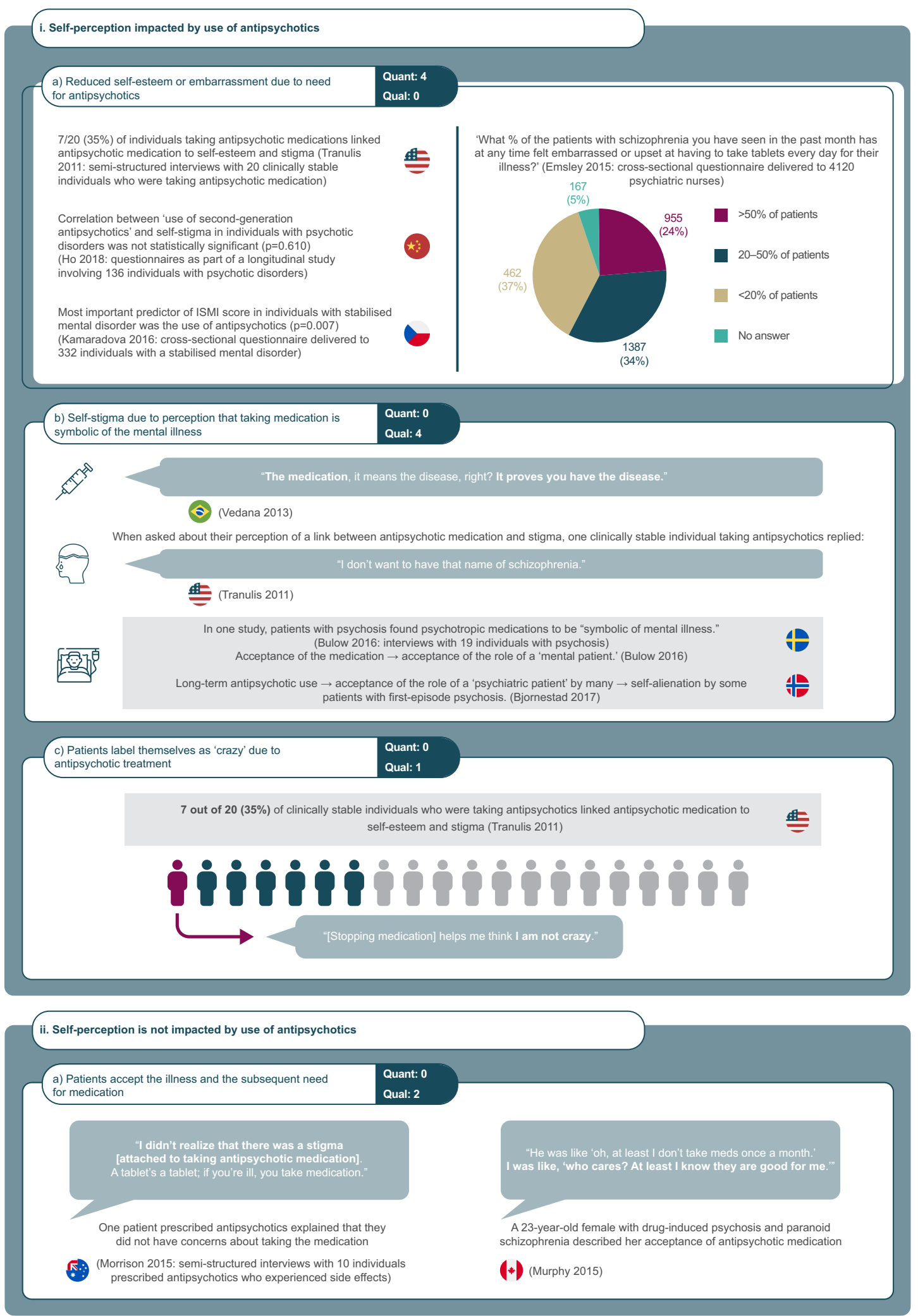

Figure 3 Qualitative and quantitative data for Theme IB: Impact of antipsychotic treatment on self-stigma. All qualitative and quantitative data relating to the impact of antipsychotic treatment on self-stigma are presented. Quotations from qualitative studies as well as quantitative results are recorded along with the study design, population, and location (where applicable; represented by country flags) for each. 

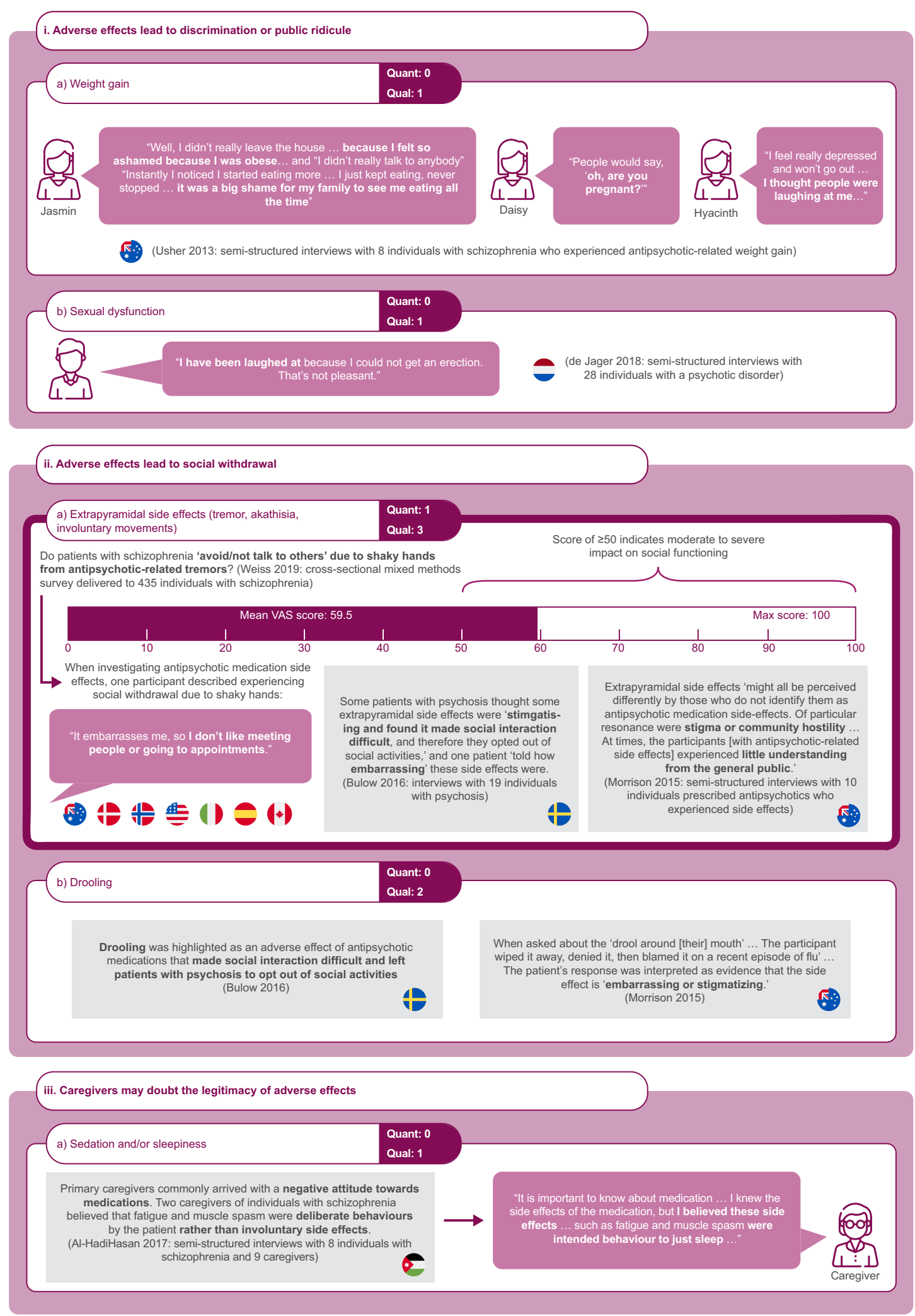

Figure 4 Qualitative and quantitative data for Theme $2 \mathrm{~A}$ : Impact of side effects of antipsychotic treatment on social stigma. All qualitative and quantitative data related to the impact of side effects of antipsychotic medications on social stigma are presented. Quotations from qualitative studies as well as quantitative results are recorded along with the study design, population, and location (where applicable; represented by country flags) for each. 


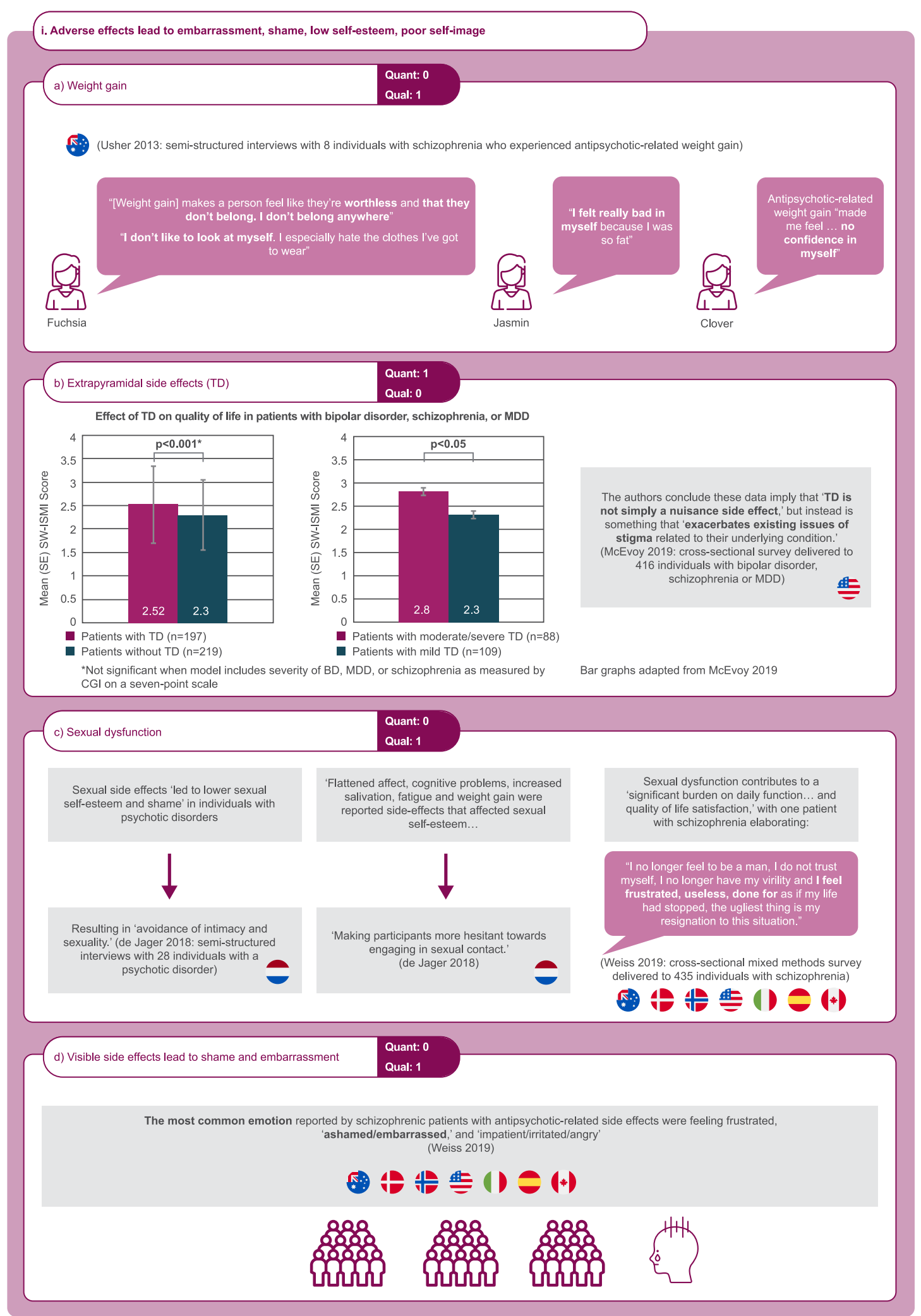

Figure 5 Qualitative and quantitative data for Theme 2B: Impact of side effects of antipsychotic treatment on self-stigma. All qualitative and quantitative data related to the impact of side effects of antipsychotic medications on self-stigma are presented. Quotations from qualitative studies as well as quantitative results are recorded along with the study design, population, and location (where applicable; represented by country flags) for each. 


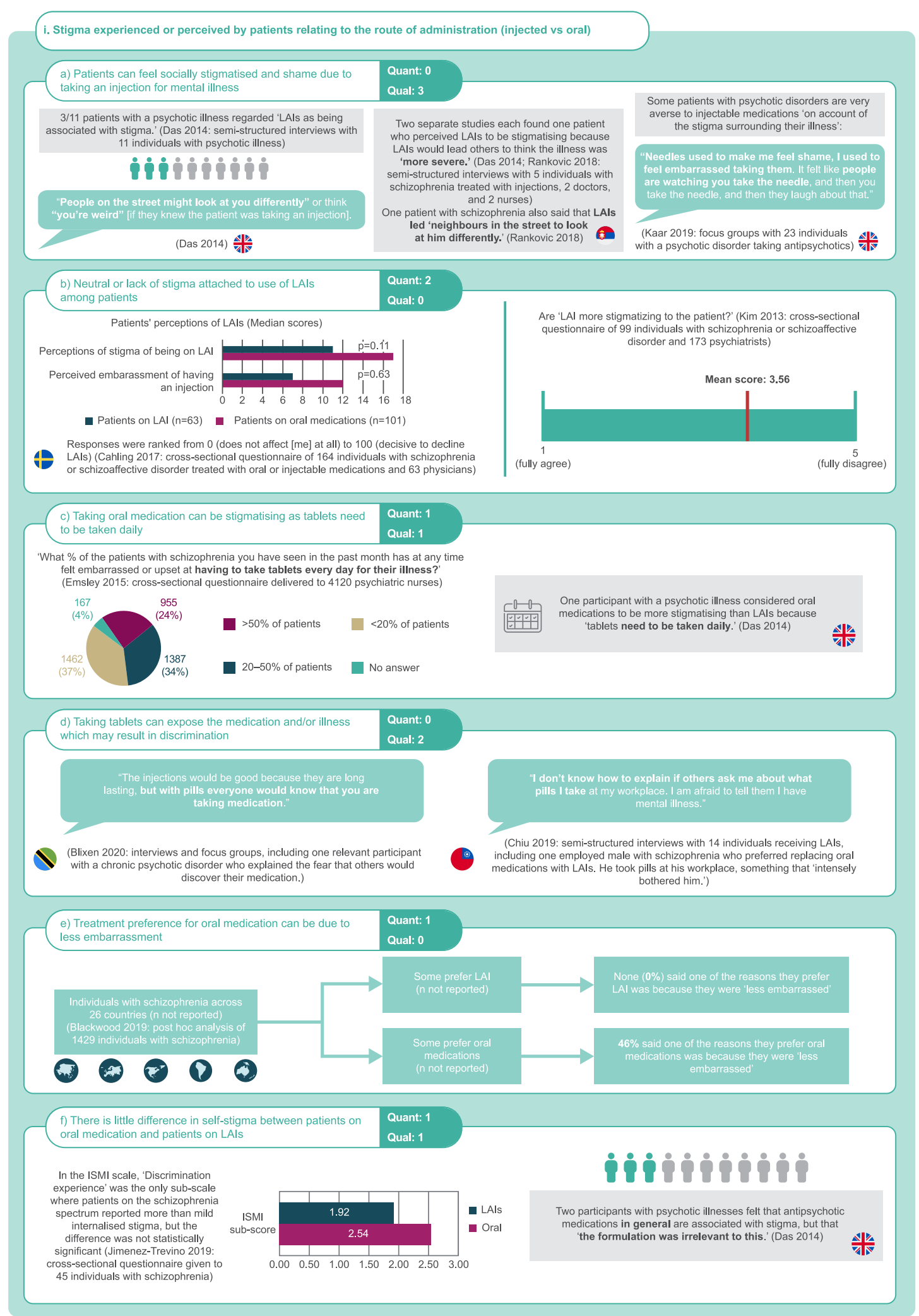

Figure 6 Qualitative and quantitative data for Theme 3: Impact of route of administration of antipsychotic treatment on stigma experienced or perceived by patients. All qualitative and quantitative data related to the impact of route of administration of antipsychotic medication are presented. Quotations from qualitative studies as well as quantitative results are recorded along with the study design, population, and location (where applicable; represented by country flags) for each. 


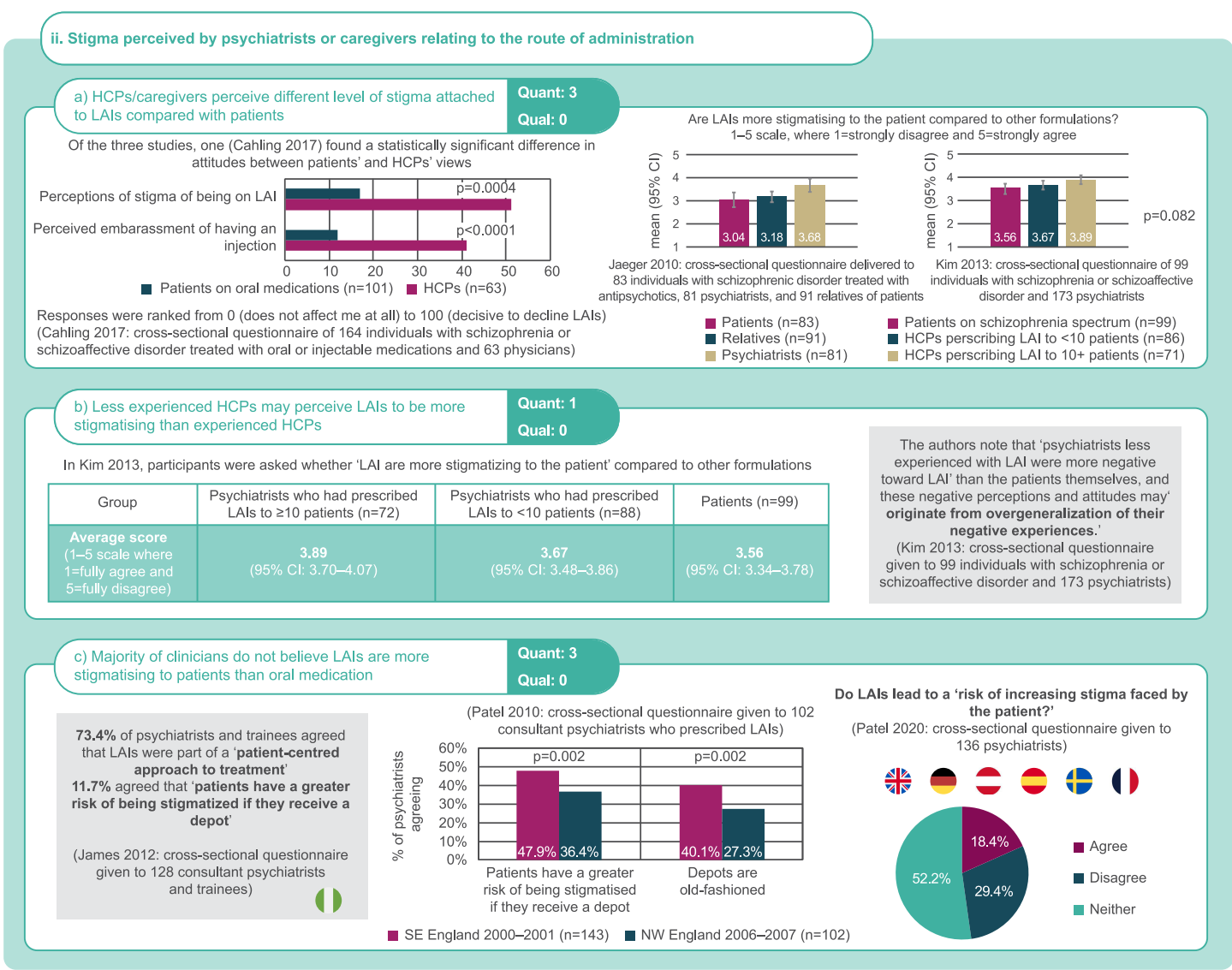

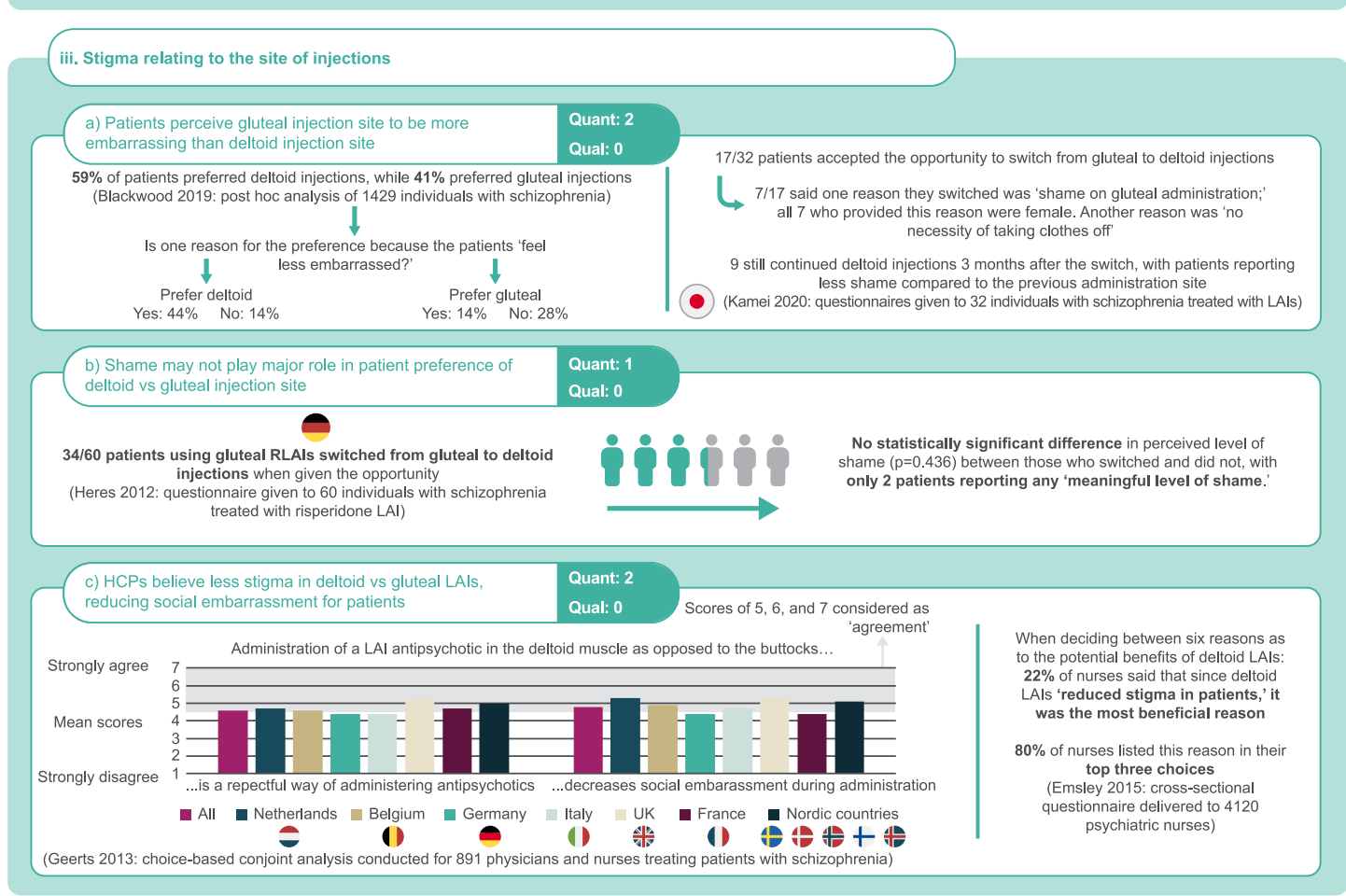

Figure 7 Qualitative and quantitative data for Theme 3: Impact of route of administration of antipsychotic treatment on stigma experienced or perceived by physicians or caregivers, or related to site of injection. All qualitative and quantitative data related to the impact of route of administration of antipsychotic medication are presented. Quotations from qualitative studies as well as quantitative results are recorded along with the study design, population, and location (where applicable; represented by country flags) for each. 


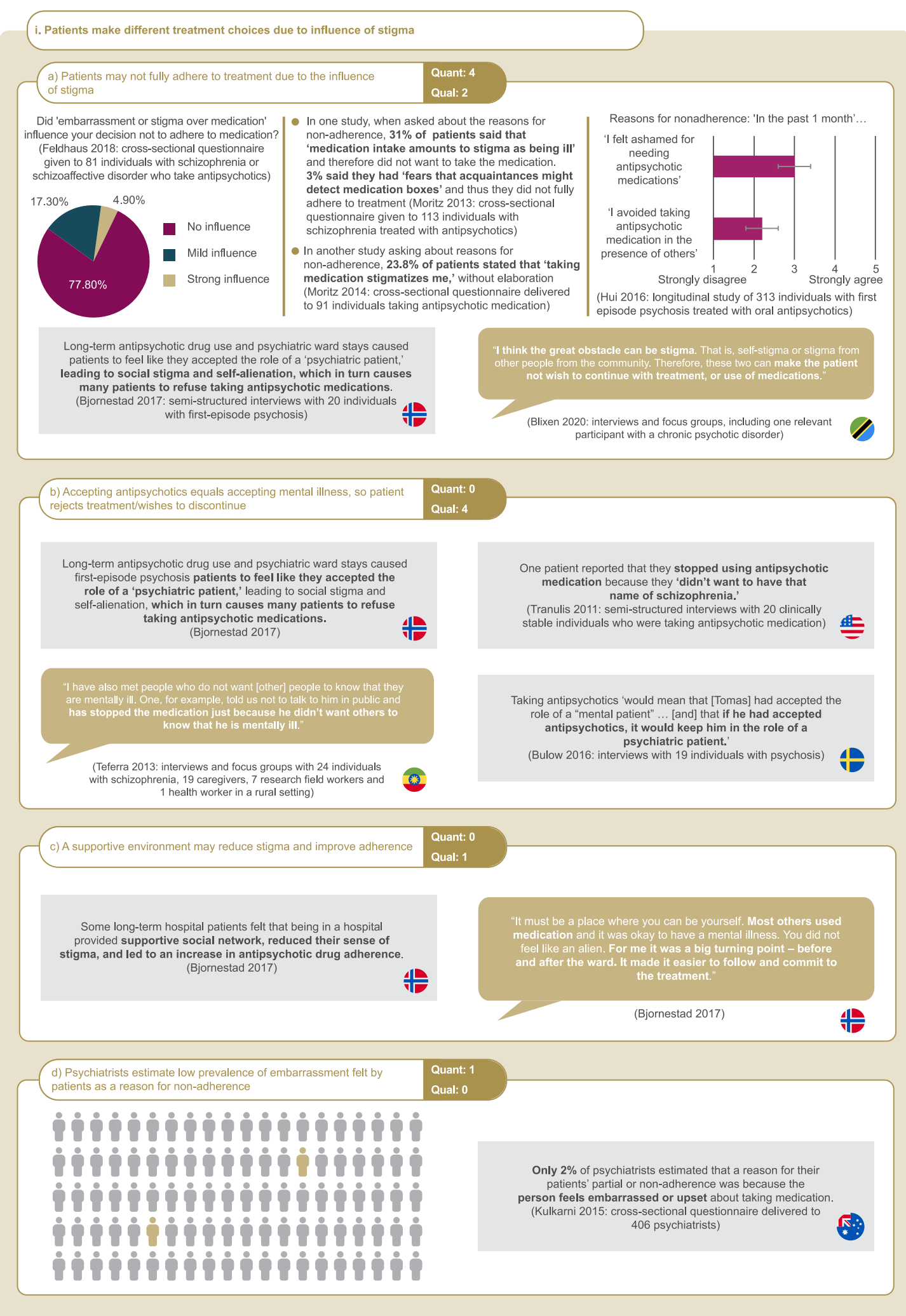

Figure 8 Qualitative and quantitative data for Theme 4: Impact of stigma on use of antipsychotics (experienced or perceived by patients). All qualitative and quantitative data related to the impact of stigma experienced or perceived by patients on the use of antipsychotics are presented. Quotations from qualitative studies as well as quantitative results are recorded along with the study design, population, and location (where applicable; represented by country flags) for each. 


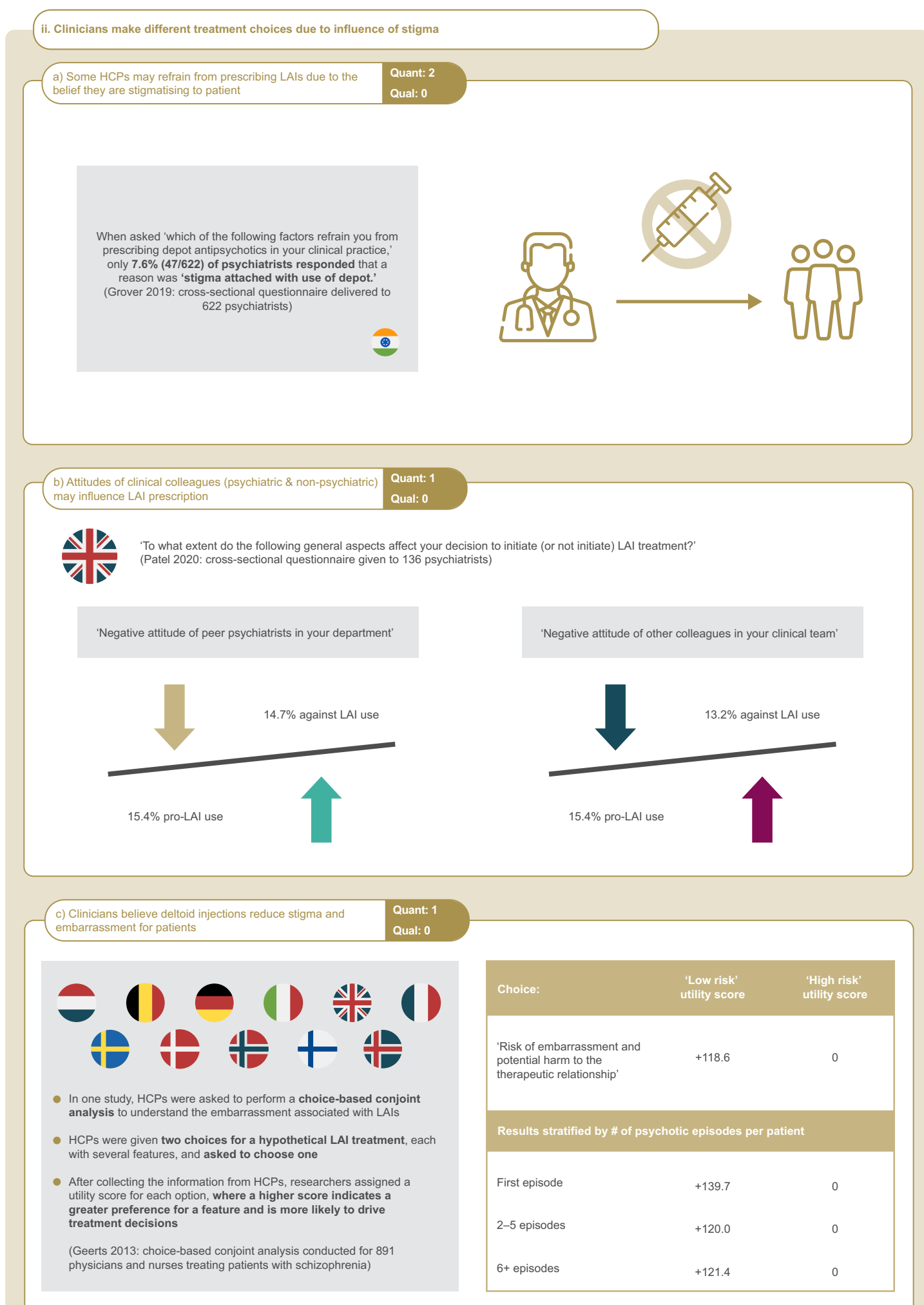

Figure 9 Qualitative and quantitative data for Theme 4: Impact of stigma on use of antipsychotics (experienced or perceived by clinicians). All qualitative and quantitative data related to the impact of stigma experienced or perceived by clinicians on the use of antipsychotics are presented. Quotations from qualitative studies as well as quantitative results are recorded along with the study design, population, and location (where applicable; represented by country flags) for each. 
$24 \%$ believed that over $50 \%$ of their patients fell into this category, $34 \%$ believed the proportion was $20-50 \%$, while $37 \%$ believed it was less than $20 \%{ }^{63}$

Several studies found that patients experienced self-stigma due to the perception that taking antipsychotic medication is symbolic of the diagnosed psychiatric condition, with one patient expressing that "the medication, it means the disease, right? It proves you have the disease".${ }^{59}$ On the other hand, certain studies also reported that some patients accepted their illness and need for medication: "I didn't realize there was a stigma [attached to taking antipsychotic medication]. A tablet's a tablet; if you're ill, you take medication." 64

\section{Theme 2A: Impact of Side Effects of Antipsychotic Treatment on Social Stigma}

Six studies reported findings on the impact of the side effects of antipsychotic medication on social stigma: five qualitative studies and one that presented both qualitative and quantitative data. All studies in this theme included patients with psychosis, with one study additionally including patients with a diagnosis of bipolar disorder. Evidence was mapped to sub-themes related to the impact of side effects of antipsychotic treatment, focusing on discrimination, social withdrawal, and caregiver perception of side effects (Figure 4).

The antipsychotic-related side effects reported in most studies as leading to discrimination or public ridicule were weight gain and sexual dysfunction, although quotations were only extracted from three studies. ${ }^{48,54,61}$ One study shared quotations from patients who self-identified as having gained weight due to antipsychotic medication and revealed a reluctance to socialise due to this weight gain: "I feel really depressed and won't go out ... I thought people were laughing at me ..." and "I didn't really leave the house ... because I felt so ashamed because I was obese ... ". ${ }^{48}$

The study containing quantitative information reported on the impact of extrapyramidal side effects (EPS) on patients' social withdrawal. The survey investigated antipsychotic side effects and found that the item "avoid/not talk to others" due to shaky hands was associated with a score of 59.5 on a visual analogue scale ranging from 0 to 100 , where a score $\geq 50$ indicates a moderate to severe impact on social functioning. ${ }^{61}$ This was supported by findings from several qualitative studies, one of which detailed how some patients with psychosis thought extrapyramidal side effects were "stigmatising and found it made social interaction difficult, and therefore they opted out of social activities." $" 37$

One study looked at the perceptions of caregivers about the legitimacy of antipsychotic side effects, and found that caregivers often have a negative attitude towards the medication and sometimes believe involuntary side effects to be deliberate behaviours by the patient. ${ }^{65}$

\section{Theme 2B: Impact of Side Effects of Antipsychotic Treatment on Self-Stigma}

Four studies reported findings on the impact of the side effects of antipsychotic medication on self-stigma: two qualitative studies, one quantitative study, and one study with both qualitative and quantitative evidence. All studies in this theme included patients with psychosis, with one study also including patients with a diagnosis of bipolar disorder or MDD. Evidence was mapped to sub-themes looking at the impact of side effects such as weight gain, sexual dysfunction, and visible side effects on patient embarrassment, shame, low self-esteem and poor self-image (Figure 5).

Weight gain and sexual dysfunction were found to play a role in self-stigma as well as social stigma, with one study reporting quotations from patients such as: "[weight gain] makes a person feel like they're worthless and that they don't belong. I don't belong anywhere", and "I felt really bad in myself because I was so fat". ${ }^{48}$ A different study found that sexual side effects "led to lower sexual self-esteem and shame" in individuals with psychiatric conditions. ${ }^{54}$

One study reported quantitative evidence examining how side effects of antipsychotics (specifically tardive dyskinesia [TD]) can lead to embarrassment, shame, and low self-esteem. The results of this cross-sectional, web-based survey suggested that the average score on the Social Withdrawal subscale of the ISMI scale (SW-ISMI) amongst patients with TD (mean: 2.52; standard deviation [SD]: 0.82) was significantly greater than that amongst patients without TD (mean 2.30; SD: 0.74; $<<0.001$ ). The authors concluded that these data imply that "TD is not simply a nuisance side effect", but instead is something that "exacerbates existing issues of stigma related to their underlying condition." 66 


\section{Theme 3: Impact of Route of Administration of Antipsychotic Treatment on Stigma}

Seventeen studies reported findings on the impact of the route of administration (oral vs long-acting injectable [LAI]) of antipsychotic medication on stigma; 12 quantitative cross-sectional studies and five qualitative interview studies. The majority of studies in this theme included patients with psychosis, with four studies focusing on HCPs with experience of prescribing antipsychotic medications, and one study including the family/caregivers of individuals with psychiatric disorders. Evidence was mapped to sub-themes examining patients' feelings of social stigma and shame due to taking an LAI or oral antipsychotic medication, stigma perceived by HCPs or caregivers relating to the route of administration of antipsychotics, and stigma relating to the injection site of LAIs (deltoid vs gluteal) (Figures 6 and 7).

Studies exploring stigma associated with LAIs versus oral medications reported a variety of responses, with some stating that patients and HCPs viewed LAIs as being less stigmatising than oral medications, and others reporting the opposite. Two studies investigating stigma related to use of LAIs specifically found that patients had a neutral response or attached a lack of stigma to LAI use. ${ }^{67,68}$ A survey of nurses across 29 countries found that $22 \%$ ranked "reduced stigma in patients" as the most beneficial reason for use of deltoid LAIs over all other forms of medication administration. The same survey found that the majority of the surveyed nurses thought that $20 \%$ or more patients with schizophrenia felt embarrassed to take tablets for their illness. ${ }^{63}$ Quotations from qualitative studies described a variety of patient opinions pertaining to route of administration, with statements from patients including: "people on the street might look at you differently" or think "you're weird" [if they knew the patient was having injections], ${ }^{69}$ and "the injections would be good because they are long lasting, but with pills everyone would know that you are taking medication." ${ }^{, 56}$ Another study asked patients if they preferred LAI or oral medications; none of the patients preferring LAIs said that their preference was because LAIs were "less embarrassing," while $46 \%$ of patients who preferred oral medications chose this reason. ${ }^{70}$ A further study comparing self-stigma between patients taking LAIs and oral medications found no significant differences in any ISMI domains between the two groups. ${ }^{71}$

Studies investigating the differing perspectives of HCPs versus patients reported similarly conflicting results. One study assessed the difference between psychiatrist and patient perceptions of LAIs, and found that psychiatrists perceive a greater amount of stigma attached to LAIs compared with patients on oral medications $(p<0.0001)$ and patients on LAIs $(p<0.0004) .{ }^{67}$ By contrast, two studies found no significant difference between patients and psychiatrists or relatives in their attitudes towards LAIs. ${ }^{68,72}$ Another study reported that psychiatrists with less experience prescribing LAIs associated LAIs with greater levels of stigma, although the differences were not statistically significant $(\mathrm{p}=0.082){ }^{68}$ Three additional studies presented quantitative evidence about stigma attached to LAIs vs oral medications by clinicians. These studies found that most clinicians do not believe LAIs are more stigmatising to patients than oral medications. $^{73-75}$

The remaining five studies reported quantitative evidence on stigma relating to the site of injection of LAIs (gluteal vs deltoid). Two studies presented evidence that patients preferred deltoid injections over gluteal injections, perceiving the deltoid site to be less embarrassing. ${ }^{47,70}$ However, another study found no statistically significant difference in perceived level of shame between a group that switched from gluteal to deltoid administration and a group that did not $(\mathrm{p}=0.436) .{ }^{46}$ Two studies found that health professionals believe deltoid injections are less stigmatising than gluteal injections. In one, $67 \%$ of surveyed psychiatric nurses agreed that "[intramuscular] administration in the deltoid muscle instead of the gluteal muscle decreases social embarrassment during administration." 76 Similarly, another study found that $22 \%$ of nurses said that deltoid LAIs "reduced stigma in patients." 63

\section{Theme 4: Impact of Stigma on Use of Antipsychotics}

Fourteen studies reported on the impact of stigma on the use of antipsychotics: five qualitative studies and nine quantitative studies. Of the 14 included studies, ten were focused on patients with psychosis, while four involved HCPs with experience of prescribing antipsychotic medications to patients with psychosis. Evidence was mapped to subthemes examining patient adherence to antipsychotics and clinician treatment choices due to the influence of stigma (Figures 8 and 9). 
Four quantitative studies suggested that embarrassment or stigma about antipsychotic medications had at least some influence on patients' adherence to treatment. ${ }^{50,51,77,78}$ Multiple qualitative studies reported similar findings, with one discussing how long-term antipsychotic drug use and psychiatric ward stays caused first-episode psychosis patients to feel like they accepted the role of a "psychiatric patient," leading to social stigma and self-alienation, which in turn caused many patients to refuse taking antipsychotic medications. ${ }^{79}$ Another study reported: "I have also met people who do not want [other] people to know that they are mentally ill. One, for example, told us not to talk to him in public and has stopped the medication just because he didn't want others to know that he is mentally ill. ${ }^{57,79,}$

One study also found that some long-term hospital patients felt that being in a hospital provided a supportive social network, reduced their sense of stigma and led to an increase in antipsychotic drug adherence. ${ }^{79}$

Two studies found evidence that some HCPs may refrain from prescribing LAIs to patients (in one study patients had psychotic disorders; in the other, both psychotic disorders and bipolar disorder) due to the belief that they are stigmatising to the patient. ${ }^{68,80}$ One study examined how the attitudes of clinical colleagues may influence LAI prescription. When asked about the "negative attitude of peer psychiatrists in [their] department," $14.7 \%$ of respondents said this motivated them against LAI use and $15.4 \%$ said that it encouraged LAI use. The physicians were also asked about the "negative attitude of other colleagues in [their] clinical team"; 13.2\% said the attitudes led them to be more anti-LAI and $15.4 \%$ said they led them to be more pro-LAI use. ${ }^{75}$ Finally, a choice-based conjoint analysis assessing how HCPs across Europe viewed LAIs found that overall, HCPs preferred a treatment where there was a low risk of damage to the therapeutic relationship, and they identified deltoid injections as having a lower risk for embarrassment than gluteal injections of LAIs. ${ }^{76}$

\section{Discussion}

This review identified and synthesised qualitative and quantitative evidence on three main sources of antipsychoticrelated stigma: perceptions of the medication itself, side effects of the medication, and route of administration. Evidence from patients and HCPs regarding the impact of stigma on the use of antipsychotics was also identified. This SLR aimed to identify evidence on stigma related to antipsychotic treatment across a range of indications. However, the vast majority of studies identified by the review focused mainly on antipsychotic treatment of patients with a diagnosis of psychosis and did not widely cover other indications, so we were not able to fulfil our objective of comparing the experiences of antipsychotic-related stigma between patients with different conditions; this remains a direction for future research.

With the emergence of COVID-19 and the subsequent lockdowns, events around the world have taken a mental and emotional toll on large sections of the population, ${ }^{81,82}$ with evidence of an increase in psychotropic medication use in the US. ${ }^{83}$ Moreover, with antipsychotic medication now approved and prescribed for a range of non-psychotic psychiatric conditions, there is a significant opportunity to help many patients around the world. ${ }^{84}$ However, as captured in this SLR, stigma surrounding antipsychotic medication still exists and serves as a barrier to acceptance and usage by patients and physicians, although the extent to which it hinders prescription and adherence, especially in patients with non-psychotic disorders, could not be determined through the included studies. By summarising the findings into four specific themes, this SLR should serve as a guide to understanding the origins of antipsychotic-related stigma in patients with psychosis and provide insights into how to address these issues. The studies included in this SLR gathered information from thousands of HCPs, caregivers, and patients across six continents, providing a global perspective and validating the applicability of the results to a variety of patient populations. For example, one study reported responses of HCPs from multiple countries to a survey regarding attitudes towards the administration of LAIs, finding that results were broadly consistent between countries. ${ }^{76}$

Findings relating to each of the four themes are discussed in detail below.

\section{Theme I: Impact of Antipsychotic Treatment on Stigma}

Theme 1 focused on the direct impact of antipsychotic treatments on social and self-stigma. Most studies from this theme were qualitative studies which explored patients' subjective experiences of antipsychotic treatment rather than focusing on stigma specifically. ${ }^{37,55-57,59,64,79}$ 
Several patients reported high levels of negative comments directed towards them because of their medication, which impacted their self-esteem. However, the most prevalent finding within this theme was that antipsychotic use was a symbol of the psychiatric condition that they had been diagnosed with. Many studies reported on individuals who felt that because they were treated with an antipsychotic medication, they were automatically considered psychiatric patients or labelled "crazy." 59,79 Patients worried about others finding out about their medication and subsequently assuming the negative stereotypes associated with people with diagnosed psychiatric disorders. ${ }^{55,56}$ Many patients were particularly worried about social stigma from people they knew, including co-workers or potential partners. ${ }^{57,79}$ The studies reporting these patient responses were conducted in multiple countries, suggesting that these fears are shared by patients on antipsychotic medication regardless of geographic location. However, one study found that with a supportive social network that minimises the risk of social stigma, individuals can feel much more comfortable with antipsychotic treatment. $^{79}$

These findings were not limited to social stigma; many studies also reported that patients had internalised these fears and considered themselves a "mental patient" while on antipsychotic medications. ${ }^{37,53,59}$ While there were a limited number of quotations supporting this sub-theme in any given study, 9/45 (20\%) of all articles included in this SLR reported on the perceived symbolism of antipsychotic medications. Furthermore, there were only two studies that reported on patients whose self-perception was not impacted by antipsychotic use. These findings highlight the importance of understanding the perspective of patients when it comes to the treatments they receive, as the way patients perceive certain medications may affect their adherence to those treatments.

\section{Theme 2: Impact of Side Effects of Antipsychotic Treatment on Stigma}

In addition to the direct impact of antipsychotics on stigma, many antipsychotic medications carry a risk of undesirable side effects, ${ }^{37,48,54,61,64}$ which can lead to external and internalised stigma. These relationships were examined in Theme 2; once again, the majority of studies provided qualitative evidence. Overall, this theme encompassed the fewest studies (eight studies reporting on social and/or self-stigma). However, it should be noted that studies had to report an explicit link between antipsychotics, side effects and stigma to be included in this SLR. For example, studies that reported stigma associated with weight gain without directly attributing this weight gain to antipsychotics were not eligible for inclusion.

Five categories of side effects were reported to have an impact on social stigma and/or self-stigma: weight gain, sexual dysfunction, EPS (tremor, akathisia, tardive dyskinesia), drooling, and sedation or sleepiness. ${ }^{37,48,54,61,64,65}$ The most commonly-reported side effects leading to stigma were weight gain and sexual dysfunction; several individuals reported on the impact of these side effects, although it should be noted that these findings were drawn from only three studies. ${ }^{48,54,61}$ In addition, EPS was commonly reported as a cause of both self-stigma and social stigma. In particular, patients reported being embarrassed by these effects, causing them to withdraw from social engagements. ${ }^{37,61,64}$

Only one study investigated how antipsychotic-related side effects were viewed by caregivers, with one relevant quotation suggesting scepticism about side effects that patients were experiencing. ${ }^{65}$ It is not possible to draw strong conclusions from this limited evidence base on caregiver perspectives. It was anticipated based on scoping of studies published before 2010 that there would be studies relating side effects of antipsychotic medication to the disclosure of patients' diagnosed psychiatric conditions, or a false perception of substance abuse resulting from side effects such as coordination problems leading to stigma. ${ }^{30}$ However, no studies reporting on these sub-themes were identified.

\section{Theme 3: Impact of Route of Administration of Antipsychotic Treatment on Stigma}

Another important facet of treatment with antipsychotic medication is the route of administration, as antipsychotics can be prescribed as either oral medication or an LAI into the deltoid or gluteal muscle. The perceptions of each method of administration can carry an associated stigma for patients. The preliminary framework included sub-themes relating to route of administration within the broader themes of social stigma or self-stigma related to antipsychotic treatment; however, due to the volume of evidence identified on this topic, a separate theme was developed to further analyse these 
data. This theme included the most studies, comprising largely quantitative results as well as a high proportion of studies reporting perspectives from clinicians. ${ }^{63,67,68,72,74-76}$

Focusing first on patients' perspectives, there were conflicting results regarding stigma associated with LAIs and oral medications. While some qualitative studies reported the perspectives of patients who found LAIs to be stigmatising, ${ }^{52,69}$ larger quantitative studies reported little to no stigma associated with LAIs. ${ }^{67,68}$ Furthermore, the studies with qualitative evidence of LAI-associated stigma only included a few relevant quotations. For oral medications, some studies suggested that the frequency and visibility of using tablets can be stigmatising, driving patients away from this option. ${ }^{56,63,69}$ However, the evidence was weak, with only three quotations from separate studies supporting this theme. A larger study reported a similar result, finding that most included patients preferred LAIs, and only a minority of patients would choose oral medications over LAIs if offered, ${ }^{70}$ but it is difficult to draw conclusions from a single study. On balance, the evidence base suggests that although some patients find LAIs stigmatising, others disagree and may be more inclined to use LAIs, partly due to the lower risk of revealing their illness to others. These conflicting findings suggest that it may be beneficial for clinicians to take individual patients' opinions on medication route of administration into account when determining the optimal course of treatment.

Three quantitative studies investigated the difference between how HCPs and patients view stigma relating to route of administration. One study reported a higher perception of LAI-associated stigma among HCPs compared to patients, and this difference was statistically significant. ${ }^{67}$ The remaining two studies did not find any statistically significant differences. ${ }^{68,72}$ Three surveys reported that a majority of clinicians do not believe LAIs are more stigmatising to patients than oral medication. ${ }^{73-75}$ Interestingly, two studies by one research group indicated that the proportion of psychiatrists who believe LAIs are more stigmatising may have decreased over time. ${ }^{74,75}$

When deciding between deltoid and gluteal injections, three studies found that just over half of patients would prefer deltoid injections to gluteal injections. ${ }^{46,47,70}$ Among these, two studies reported that embarrassment was the reason for a moderate proportion of patients ( $\sim 40 \%$ in both studies) ${ }^{47,70}$ while the third reported only a small proportion of patients with a "meaningful level of shame". ${ }^{46}$ Another study noted that the patients who reported less shame with deltoid injections were all women ${ }^{47}$ it may therefore be relevant for future studies to investigate gender-based differences in antipsychotic-related stigma. Two international surveys of nurses reported that the majority of nurses agreed with statements indicating that deltoid injections reduce stigma. ${ }^{63,76}$

In the preliminary framework, it was anticipated that some studies would link the perceived coerciveness of LAIs with stigma. Although some of the screened studies did mention that LAIs are perceived as coercive, they did not expand further on this concept or report a direct link to stigma.

\section{Theme 4: Impact of Stigma on Use of Antipsychotics}

The three aforementioned themes focused on the perceptions and viewpoints of patients, HCPs and caregivers. Theme 4, however, focused on actions - do patients and clinicians act differently based on the views reported?

When examining responses of patients, many studies reported that some patients will take medications less frequently due to stigma attached to medication or the perceived association between antipsychotic medication and diagnosed psychiatric conditions. ${ }^{50,51,77-79}$ However, none of the studies reported how many patients out of a total sample refused to take antipsychotic medication due to stigma. Rather, the studies sought out patients who were non-adherent and investigated the reasons. Among quantitative studies, the proportion of patients self-reporting stigma as a cause of nonadherence varied, while the heterogeneity in study design and survey response options made it challenging to compare the results directly.

Only four studies explored the impact of stigma on clinicians' treatment decisions, all focusing on LAIs. ${ }^{74-76,80}$ No studies were found to explore whether clinicians consider the wider stigma associated with antipsychotics or the risk of stigmatising side effects when deciding which antipsychotic (if any) to prescribe. The studies suggest that some psychiatrists may be less inclined to administer LAIs due to perceived patient stigma and disapproval from colleagues. ${ }^{75}$ Moreover, some physicians reported that they may be less inclined to prescribe LAIs, particularly gluteal LAIs, because they place a strong emphasis on the therapeutic relationship and do not want to cause embarrassment for the patient. ${ }^{76}$ 


\section{Strengths and Limitations}

This review used systematic methods consistent with the Cochrane Handbook for Systematic Reviews of Interventions to conduct an exhaustive search of the literature. This SLR also employed an adaptation of framework synthesis, which allowed for the synthesis of heterogeneous data from diverse sources and contexts, including both quantitative and qualitative evidence. Although methods for synthesising these heterogeneous data types are not yet welldeveloped, the risk of misinterpreting data was minimised by only extracting data of firm relevance to stigma related to antipsychotic medications. Furthermore, this review was designed to explore themes rather than to test hypotheses, so the benefits of the varied evidence base outweigh the disadvantages. Independent data extraction and synthesis allowed for the emergence of novel themes from the identified data, and reinforced the interpretations made by the reviewers. Only primary research studies published since 2010 were eligible for this SLR, ensuring only contemporary data were included, although this may have resulted in some perspectives on antipsychotic-related stigma being missed.

This review focused specifically on stigma related to antipsychotic medication, so studies with tenuous links between stigma and antipsychotic medications were excluded. For example, a statement like "patients want to avoid antipsychotic-related weight gain" was not included unless the study explicitly linked this to social or self-stigma. Searches were both language- and date-limited which may have excluded some relevant information and limited the global applicability of the findings. Additionally, the vast majority of studies identified by the review focused on patients with psychotic disorders, and none presented separate results for patients with non-psychotic conditions. Therefore, this SLR was unable to establish whether the extent and experience of antipsychotic-related stigma differs between patients with psychotic disorders and non-psychotic disorders like MDD. This topic should be explored in future research. It should also be noted that data were obtained from studies conducted in a wide range of countries; therefore, some differences between studies may be related to cultural and regional variations in the way stigma is experienced and reported.

Across themes, studies reporting quantitative evidence generally reported a low prevalence of stigma associated with antipsychotic medications, while the qualitative evidence demonstrated that some patients do experience stigma. This discrepancy might be due to the study methodology, with qualitative studies often taking place as interviews or group discussions in which the subject of stigma was purposely explored. Qualitative evidence was often limited, with some studies providing only a small number of relevant quotations for extraction. As a result, it is difficult to know the extent to which this evidence reflects a wider patient population. However, the quotations serve to illustrate the themes and illuminate the treatment experience of certain patients, indicating that there is a substantial burden of stigma for a number of patients and that this impacts their adherence. The qualitative data identified in this SLR provide context and an improved understanding of how stigma arises and is perpetuated among patients and HCPs.

\section{Conclusions}

To our knowledge, this is the first SLR to focus specifically on stigma associated with antipsychotic medication. Using framework synthesis, we were able to synthesise evidence from both quantitative and qualitative studies and include diverse perspectives from patients, caregivers and clinicians. Although there are limited data on the overall prevalence of antipsychotic-related stigma and its impact on the use of antipsychotics, the evidence identified by this SLR demonstrates that a number of patients do experience or perceive direct stigma (such as being labelled "crazy" due to use of antipsychotics) and indirect stigma (such as ridicule due to antipsychotic-related weight gain), and that this is a factor in non-adherence to antipsychotic medication.

Further research could focus on the key evidence gaps identified in this SLR, such as the prevalence and experience of antipsychotic-associated stigma in patients with non-psychotic conditions who are eligible for treatment with antipsychotics, patient characteristics that might impact stigma (such as gender), the prevalence of stigma-related noncompliance in a wider sample of patients that is not limited to non-adherent patients, and the extent to which clinicians take into account the potential stigma associated with antipsychotic treatments or side effects of different antipsychotics when making treatment decisions for patients with psychotic or non-psychotic conditions. 


\section{Abbreviations}

APA, American Psychiatric Association; CANMAT, Canadian Network for Mood and Anxiety Treatments; CDSR, Cochrane Database of Systematic Reviews; CENTRAL, Cochrane Central Register of Controlled Trials; DARE, Database of Abstracts of Reviews of Effects; ENTREQ, Enhancing Transparency in Reporting the Synthesis of Qualitative Research; EPS, extrapyramidal side effects; GABA, gamma aminobutyric acid; HCP, healthcare provider; ISMI, Internalised Stigma of Mental Illness; LAI, long-acting injectable; MeSH, Medical Subject Heading; MDD, major depressive disorder; NICE, National Institute for Health and Care Excellence; PTSD, post-traumatic stress disorder; SLR, systematic literature review; SPIDER, Sample, Phenomenon of Interest, Design, Evaluation, Research type; SW-ISMI, Social Withdrawal subscale of the ISMI scale; TD, tardive dyskinesia.

\section{Data Sharing Statement}

This study is an SLR and no novel data were generated. All data relevant to the study are either included in the article or uploaded as supplementary information.

\section{Acknowledgments}

The authors acknowledge Aaditya Rawal, BS, MSL, from Costello Medical, USA, for support with conducting the SLR; Isabel Haber, BS, from Costello Medical, USA, for medical writing and editorial assistance based on the authors' input and direction; and Fay Angel, BA (Hons), from Costello Medical, UK, for graphic design support.

\section{Author Contributions}

All authors made a significant contribution to the work reported, whether that is in the conception, study design, execution, acquisition of data, analysis and interpretation, or in all these areas; took part in drafting, revising or critically reviewing the article; gave final approval of the version to be published; have agreed on the journal to which the article has been submitted; and agree to be accountable for all aspects of the work.

\section{Funding}

This study was sponsored by Otsuka Pharmaceutical Development \& Commercialization Inc. and H. Lundbeck A/S, including support for third-party writing assistance for this article, provided by Isabel Haber, BS, Costello Medical, USA, in accordance with Good Publication Practice (GPP3) guidelines (http://www.ismpp.org/gpp3).

\section{Disclosure}

MT: Received consulting fees from Otsuka; KP: Employee of Otsuka Pharmaceutical Development \& Commercialization, Inc.; ABH: Employee of Costello Medical, Inc., which received consulting fees from Otsuka for this review; EW: Employee of Costello Medical, Ltd., which received consulting fees from Otsuka for this review; DP: Employee of Costello Medical, Ltd., which received consulting fees from Otsuka for this review; MB: Employee of Otsuka Pharmaceutical Development \& Commercialization, Inc.; CH: Employee of Lundbeck LLC; TNM: Employee of Lundbeck LLC; HW: Employee of Otsuka Pharmaceutical Development \& Commercialization, Inc. The authors report no other conflicts of interest in this work.

\section{References}

1. American Psychiatric Association. Diagnostic and Statistical Manual of Mental Disorders. 5th ed. 2013.

2. Ripoll LH. Psychopharmacologic treatment of borderline personality disorder. Dialogues Clin Neurosci. 2013;15(2):213-224. doi:10.31887/ DCNS.2013.15.2/lripoll

3. Nelson JC, Weiller E, Zhang P, Weiss C, Hobart M. Efficacy of adjunctive brexpiprazole on the core symptoms of major depressive disorder: a post hoc analysis of two pooled clinical studies. $J$ Affect Disord. 2018;227:103-108. doi:10.1016/j.jad.2017.09.054

4. Villarreal G, Hamner MB, Qualls C, Cañive JM. Characterizing the effects of quetiapine in military post-traumatic stress disorder. Psychopharmacol Bull. 2018;48(2):8-17.

5. Durgam S, Earley W, Guo H, et al. Efficacy and safety of adjunctive cariprazine in inadequate responders to antidepressants: a randomized, double-blind, placebo-controlled study in adult patients with major depressive disorder. J Clin Psychiatry. 2016;77(3):371-378. doi:10.4088/ JCP. $15 \mathrm{~m} 10070$ 
6. Timäus C, Meiser M, Bandelow B, et al. Pharmacotherapy of borderline personality disorder: what has changed over two decades? A retrospective evaluation of clinical practice. BMC Psychiatry. 2019;19(1):393. doi:10.1186/s12888-019-2377-z

7. Wang P, Si T. Use of antipsychotics in the treatment of depressive disorders. Shanghai Arch Psychiatry. 2013;25(3):134-140. doi:10.3969/j. issn.1002-0829.2013.03.002

8. Association AP. Practice guidelines for the treatment of patients with major depressive disorder; 2010.

9. Kennedy SH, Lam RW, McIntyre RS, et al. Canadian Network for Mood and Anxiety Treatments (CANMAT) 2016 clinical guidelines for the management of adults with major depressive disorder: section 3. Pharmacological treatments. Can J Psychiatry. 2016;61(9):540-560. doi:10.1177/ 0706743716659417

10. Health NCCfM. Depression: the treatment and management of depression in adults. National Institute for Health and Clinical Excellence; 2009.

11. Therapeutics Initiative: Evidence Based Drug Therapy. Antipsychotics should not be used for non-psychotic depression. Available from: https:// www.ti.ubc.ca/2015/09/30/antipsychotics-should-not-be-used-for-non-psychotic-depression/. Accessed January 19, 2022.

12. Sheehan R, Hassiotis A, Walters K, Osborn D, Strydom A, Horsfall L. Mental illness, challenging behaviour, and psychotropic drug prescribing in people with intellectual disability: UK population based cohort study. BMJ. 2015;351:h4326. doi:10.1136/bmj.h4326

13. Komossa K, Depping AM, Gaudchau A, Kissling W, Leucht S. Second-generation antipsychotics for major depressive disorder and dysthymia. Cochrane Database Syst Rev. 2010;(12):Cd008121. doi:10.1002/14651858.CD008121.pub2

14. Mulder R, Hamilton A, Irwin L, et al. Treating depression with adjunctive antipsychotics. Bipolar Disord. 2018;20(S2):17-24. doi:10.1111/ bdi. 12701

15. Haddad PM, Brain C, Scott J. Nonadherence with antipsychotic medication in schizophrenia: challenges and management strategies. Patient Relat Outcome Meas. 2014;5:43-62. doi:10.2147/PROM.S42735

16. Semahegn A, Torpey K, Manu A, Assefa N, Tesfaye G, Ankomah A. Psychotropic medication non-adherence and its associated factors among patients with major psychiatric disorders: a systematic review and meta-analysis. Syst Rev. 2020;9(1):17. doi:10.1186/s13643-020-1274-3

17. Cramer JA, Rosenheck R. Compliance with medication regimens for mental and physical disorders. Psychiatr Serv. 1998;49(2):196-201. doi:10.1176/ps.49.2.196

18. Lacro JP, Dunn LB, Dolder CR, Leckband SG, Jeste DV. Prevalence of and risk factors for medication nonadherence in patients with schizophrenia: a comprehensive review of recent literature. J Clin Psychiatry. 2002;63(10):892-909. doi:10.4088/JCP.v63n1007

19. Pousa E, Ochoa S, Cobo J, et al. A deeper view of insight in schizophrenia: insight dimensions, unawareness and misattribution of particular symptoms and its relation with psychopathological factors. Schizophr Res. 2017;189:61-68. doi:10.1016/j.schres.2017.02.016

20. Hudson TJ, Owen RR, Thrush CR, et al. A pilot study of barriers to medication adherence in schizophrenia. J Clin Psychiatry. 2004;65(2):211-216. doi:10.4088/JCP.v65n0211

21. McCann TV, Boardman G, Clark E, Lu S. Risk profiles for non-adherence to antipsychotic medications. J Psychiatr Ment Health Nurs. 2008;15 (8):622-629. doi:10.1111/j.1365-2850.2008.01277.x

22. Rummel-Kluge C, Schuster T, Peters S, Kissling W. Partial compliance with antipsychotic medication is common in patients with schizophrenia. Aust. N Z J Psychiatry. 2008;42(5):382-388. doi:10.1080/00048670801961107

23. Brain C, Sameby B, Allerby K, et al. Stigma, discrimination and medication adherence in schizophrenia: results from the Swedish COAST study. Psychiatry Res. 2014;220(3):811-817. doi:10.1016/j.psychres.2014.10.016

24. Kamaradova D, Latalova K, Prasko J, et al. Connection between self-stigma, adherence to treatment, and discontinuation of medication. Patient Prefer Adherence. 2016;10:1289-1298. doi:10.2147/PPA.S99136

25. Liu-Seifert H, Adams DH, Ascher-Svanum H, Faries DE, Kinon BJ. Patient perception of medication benefit and early treatment discontinuation in a 1-year study of patients with schizophrenia. Patient Prefer Adherence. 2007;1:9-17.

26. Vrbová K, Kamarádová D, Látalová K, et al. Self-stigma and adherence to medication in patients with psychotic disorders-cross-sectional study. Neuro Endocrinol Lett. 2014;35(7):645-652.

27. Morken G, Widen JH, Grawe RW. Non-adherence to antipsychotic medication, relapse and rehospitalisation in recent-onset schizophrenia. $B M C$ Psychiatry. 2008;8:32. doi:10.1186/1471-244X-8-32

28. El-Mallakh P, Findlay J. Strategies to improve medication adherence in patients with schizophrenia: the role of support services. Neuropsychiatr Dis Treat. 2015;11:1077-1090. doi:10.2147/NDT.S56107

29. Corrigan PW, Druss BG, Perlick DA. The impact of mental illness stigma on seeking and participating in mental health care. Psychol Sci Public Interest. 2014;15(2):37-70. doi:10.1177/1529100614531398

30. Novak L, Svab V. Antipsychotics side effects' influence on stigma of mental illness: focus group study results. Psychiatr Danub. 2009;21 (1):99-102.

31. Sajatovic M, Jenkins JH. Is antipsychotic medication stigmatizing for people with mental illness? Int Rev Psychiatry. 2007;19(2):107-112. doi:10.1080/09540260701278911

32. Brohan E, Henderson C, Wheat K, et al. Systematic review of beliefs, behaviours and influencing factors associated with disclosure of a mental health problem in the workplace. BMC Psychiatry. 2012;12:11. doi:10.1186/1471-244x-12-11

33. Gronholm PC, Thornicroft G, Laurens KR, Evans-Lacko S. Mental health-related stigma and pathways to care for people at risk of psychotic disorders or experiencing first-episode psychosis: a systematic review. Psychol Med. 2017;47(11):1867-1879. doi:10.1017/S0033291717000344

34. Parcesepe AM, Cabassa LJ. Public stigma of mental illness in the United States: a systematic literature review. Adm Policy Ment Health. 2013;40 (5):384-399. doi:10.1007/s10488-012-0430-z

35. Schnyder N, Panczak R, Groth N, Schultze-Lutter F. Association between mental health-related stigma and active help-seeking: systematic review and meta-analysis. Br J Psychiatry. 2018;210(4):261-268. doi:10.1192/bjp.bp.116.189464

36. Büchter RB, Messer M. Interventions for reducing self-stigma in people with mental illnesses: a systematic review of randomized controlled trials. GMS e-Journal. 2017;15:Doc07. doi:10.3205/000248

37. Bulow P, Andersson G, Denhov A, Topor A. Experience of psychotropic medication -an interview study of persons with psychosis. Issues Mental Health Nurs. 2016;37(11):820-828. doi:10.1080/01612840.2016.1224283

38. Tong A, Flemming K, McInnes E, Oliver S, Craig J. Enhancing transparency in reporting the synthesis of qualitative research: ENTREQ. BMC Med Res Methodol. 2012;12(1):181. doi:10.1186/1471-2288-12-181 
39. Cooke A, Smith D, Booth A. Beyond PICO: the SPIDER tool for qualitative evidence synthesis. Qual Health Res. 2012;22(10):1435-1443. doi:10.1177/1049732312452938

40. Booth A, Carroll C. How to build up the actionable knowledge base: the role of "best fit" framework synthesis for studies of improvement in healthcare. BMJ Qual Saf. 2015;24(11):700-708. doi:10.1136/bmjqs-2014-003642

41. Khan MS, Walter T, Buchanan-Hughes A, et al. Differential diagnosis of diarrhoea in patients with neuroendocrine tumours: a systematic review. World J Gastroenterol. 2020;26(30):4537-4556. doi:10.3748/wjg.v26.i30.4537

42. Asghari S, Hurd J, Marshall Z, et al. Challenges with access to healthcare from the perspective of patients living with HIV: a scoping review \& framework synthesis. AIDS Care. 2018;30(8):963-972. doi:10.1080/09540121.2018.1435848

43. Centre for Reviews and Dissemination. Systematic Reviews: CRD's Guidance for Undertaking Reviews in Health Care. University of York: Centre for Reviews and Dissemination; 2008.

44. Joanna Briggs Institute. Critical appraisal tools. Available from: https://joannabriggs.org/critical-appraisal-tools. Accessed January $19,2022$.

45. Beel J, Gipp B, Langer S, Genzmehr M. Docear: an academic literature suite for searching, organizing and creating academic literature. presented at: Proceedings of the 11th annual international ACM/IEEE joint conference on Digital libraries; 2011; Ottawa, Ontario, Canada. doi:10.1145/ 1998076.1998188.

46. Heres S, Frobose T, Hamann J, et al. Patients' acceptance of the deltoid application of risperidone long-acting injection. Eur Neuropsychopharmacol. 2012;22(12):897-901. doi:10.1016/j.euroneuro.2012.04.014

47. Kamei H, Homma Y, Takeuchi I, et al. Acceptance of the deltoid muscle injection of aripiprazole long-acting injectable in the patients with schizophrenia. Clin. 2020;18(1):49-57. doi:10.9758/cpn.2020.18.1.49

48. Usher K, Park T, Foster K. The experience of weight gain as a result of taking second-generation antipsychotic medications: the mental health consumer perspective. J Psychiatr Ment Health Nurs. 2013;20(9):801-806. doi:10.1111/jpm.12019

49. Lau KC, Lee EH, Hui CL, Chang WC, Chan SK, Chen EY. Psychosis patients' knowledge, adherence and attitudes towards the naming of antipsychotic medication in Hong Kong. Early Interv Psychiatry. 2015;9(5):422-427. doi:10.1111/eip.12169

50. Moritz S, Favrod J, Andreou C, et al. Beyond the usual suspects: positive attitudes towards positive symptoms is associated with medication noncompliance in psychosis. Schizophr Bull. 2013;39(4):917-922. doi:10.1093/schbul/sbs005

51. Moritz S, Hunsche A, Lincoln TM. Nonadherence to antipsychotics: the role of positive attitudes towards positive symptoms. Eur Neuropsychopharmacol. 2014;24(11):1745-1752. doi:10.1016/j.euroneuro.2014.09.008

52. Kaar SJ, Gobjila C, Butler E, Henderson C, Howes OD. Making decisions about antipsychotics: a qualitative study of patient experience and the development of a decision aid. BMC Psychiatry. 2019;19(1):309. doi:10.1186/s12888-019-2304-3

53. Tranulis C, Goff D, Henderson DC, Freudenreich O. Becoming adherent to antipsychotics: a qualitative study of treatment-experienced schizophrenia patients. Psychiatr Serv. 2011;62(8):888-892. doi:10.1176/ps.62.8.pss6208_0888

54. de Jager J, van Greevenbroek R, Nugter A, van os J. Sexual expression and it's determinants, in people diagnosed with psychotic disorders. Community Ment Health J. 2018;54(7):1082-1088. doi:10.1007/s10597-018-0285-1

55. Morant N, Azam K, Johnson S, Moncrieff J. The least worst option: user experiences of antipsychotic medication and lack of involvement in medication decisions in a UK community sample. J Ment Health. 2018;27(4):322-328. doi:10.1080/09638237.2017.1370637

56. Blixen C, Lema I, Mbwambo J, Kaaya S, Levin JB, Sajatovic M. Community perceptions of barriers to management of chronic psychotic disorders and knowledge and attitudes about long-acting injectable antipsychotic medication: qualitative study in Dar Es Salaam, Tanzania. BJPsych Open. 2020;6(2):e27. doi:10.1192/bjo.2020.4

57. Teferra S, Hanlon C, Beyero T, Jacobsson L, Shibre T. Perspectives on reasons for non-adherence to medication in persons with schizophrenia in Ethiopia: a qualitative study of patients, caregivers and health workers. BMC Psychiatry. 2013;13:168. doi:10.1186/1471-244X-13-168

58. Chiu LL, Liu CH, Chu CL, Lin HL, Lii SC. Patients' experiences of long-acting injectable antipsychotics: a qualitative study. Neuropsychiatr Dis Treat. 2019;15:1797-1804. doi:10.2147/ndt.s206154

59. Vedana K, Zanetti A. Acting for relief: coping with schizophrenia and nuisances caused by drug treatment. Ciênc Cuid Saúde. 2013;12:365-374.

60. Murphy AL, Gardner DM, Kisely S, et al. Study of antipsychotic medication experiences of youth. J Can Acad Child Adolesc Psychiatry. 2015;24 (1):61-69.

61. Weiss C. The impact of second-generation antipsychotic side-effects on functioning from a schizophrenia patient perspective: a global patient centered survey. presented at: Psych Congress; 2019; 2019.

62. Ho RWH, Chang WC, Kwong VWY, et al. Prediction of self-stigma in early psychosis: 3-Year follow-up of the randomized-controlled trial on extended early intervention. Schizophr Res. 2018;195:463-468. doi:10.1016/j.schres.2017.09.004

63. Emsley R, Alptekin K, Azorin JM, et al. Nurses' perceptions of medication adherence in schizophrenia: results of the ADHES cross-sectional questionnaire survey. Ther Adv Psychopharmacol. 2015;5(6):339-350. doi:10.1177/2045125315612013

64. Morrison P, Meehan T, Stomski NJ. Living with antipsychotic medication side-effects: the experience of Australian mental health consumers. Int J Ment Health Nurs. 2015;24(3):253-261. doi:10.1111/inm.12110

65. Al-HadiHasan A, Callaghan P, Lymn JS. Qualitative process evaluation of a psycho-educational intervention targeted at people diagnosed with schizophrenia and their primary caregivers in Jordan. Journal Article; Randomized Controlled Trial. BMC Psychiatry. 2017;17(1):68. doi:10.1186/ s12888-017-1225-2

66. McEvoy J, Gandhi SK, Rizio AA, et al. Effect of tardive dyskinesia on quality of life in patients with bipolar disorder, major depressive disorder, and schizophrenia. Qual Life Res. 2019;28(12):3303-3312. doi:10.1007/s11136-019-02269-8

67. Cahling L, Berntsson A, Broms G, Ohrmalm L. Perceptions and knowledge of antipsychotics among mental health professionals and patients. BJPsych Bulletin. 2017;41(5):254-259. doi:10.1192/pb.bp.116.055483

68. Kim SW, Lee YH, Jang JE, et al. Comparison of attitudes toward long-acting injectable antipsychotics among psychiatrists and patients. Comparative Study Multicenter Study Research Support, Non-U.S. Gov't. Int Clin Psychopharmacol. 2013;28(2):80-86. doi:10.1097/ YIC.0b013e32835d30ae

69. Das AK, Malik A, Haddad PM. A qualitative study of the attitudes of patients in an early intervention service towards antipsychotic long-acting injections. Ther Adv Psychopharmacol. 2014;4(5):179-185. doi:10.1177/2045125314542098

70. Blackwood C, Sanga P, Nuamah I, et al. Patients' preference for long-acting injectable versus oral antipsychotics in schizophrenia: results from the patient-reported medication preference questionnaire. Patient Prefer Adherence. 2020;14:1093-1102. doi:10.2147/ppa.s251812 
71. Jimenez-Trevino L, Garcia JC, Sanchez MAP, et al. Self stigma in patients with schizophrenia spectrum disorders: oral versus LAI antipsychotic pharmacological approaches. presented at: APA; 2019; 2019.

72. Jaeger M, Rossler W. Attitudes towards long-acting depot antipsychotics: a survey of patients, relatives and psychiatrists. Research Support, NonU.S. Gov’t. Psychiatry Res. 2010;175(1-2):58-62. doi:10.1016/j.psychres.2008.11.003

73. James BO, Omoaregba JO, Okonoda KM, Otefe EU, Patel MX. The knowledge and attitudes of psychiatrists towards antipsychotic long-acting injections in Nigeria. Ther Adv Psychopharmacol. 2012;2(5):169-177. doi:10.1177/2045125312453158

74. Patel MX, Haddad PM, Chaudhry IB, McLoughlin S, David AS. Psychiatrists' use, knowledge and attitudes to first- and second-generation antipsychotic long-acting injections: comparisons over 5 years. J Psychopharmacol. 2010;24(10):1473-1482. doi:10.1177/0269881109104882

75. Patel MX, Bent-Ennakhil N, Sapin C, et al. Attitudes of European physicians towards the use of long-acting injectable antipsychotics. BMC Psychiatry. 2020;20(1):123. doi:10.1186/s12888-020-02530-2

76. Geerts P, Martinez G, Schreiner A. Attitudes towards the administration of long-acting antipsychotics: a survey of physicians and nurses. BMC Psychiatry. 2013;13(58). doi:10.1186/1471-244X-13-58

77. Feldhaus T, Falke S, von Gruchalla L, et al. The impact of self-stigmatization on medication attitude in schizophrenia patients. Psychiatry Res. 2018;261:391-399. doi:10.1016/j.psychres.2018.01.012

78. Hui CLM, Poon VWY, Ko WT, et al. Risk factors for antipsychotic medication non-adherence behaviors and attitudes in adult-onset psychosis. Schizophr Res. 2016;174(1-3):144-149. doi:10.1016/j.schres.2016.03.026

79. Bjornestad J, Davidson L, Joa I, et al. Antipsychotic treatment: experiences of fully recovered service users. J Mental Health. 2017;26(3):264-270. doi:10.1080/09638237.2017.1294735

80. Grover S, Sahoo S, Mehra A. Perceptions of psychiatrists toward the use of long-acting injectable antipsychotics: an online survey study from India. J Clin Psychopharmacol. 2019;39(6):611-619. doi:10.1097/JCP.0000000000001109

81. Vindegaard NM. COVID-19 pandemic and mental health consequences: systematic review of the current evidence. Brain Behav Immun. 2020;89:531-542. doi:10.1016/j.bbi.2020.05.048

82. Barger LK, Czeisler CA, Howard ME, Shantha MW. Mental health, substance use, and suicidal ideation during the COVID-19 pandemic. MMWR Morb Mortal Wkly Rep. 2020;69:1049-1057. doi:10.15585/mmwr.mm6932a1

83. Express Scripts. U.S. trends in medication use for depression, anxiety and insomnia. America's state of mind; 2020.

84. Wen XJ, Wang LM, Liu ZL, Huang A, Liu YY, Hu JY. Meta-analysis on the efficacy and tolerability of the augmentation of antidepressants with atypical antipsychotics in patients with major depressive disorder. Braz J Med Biol. 2014;47(7):605-616. doi:10.1590/1414-431x20143672

85. Kulkarni RR. Low-dose amisulpride for debilitating clozapine-induced sialorrhea: case series and review of literature. Indian J Psychol Med. 2015;37:446-448. doi:10.4103/0253-7176.168592

86. Noor Us Saba Z, Muraraiah S, Chandrashekar H. Medication adherence in schizophrenia: understanding patient's views. Natl J Physiol Pharm Pharmacol. 2019;9:373-378.

87. Rankovic A, Jankovic S. Problems with the administration of antipsychotic drugs in depot formulations in the treatment of schizophrenia. Acta Fac Med Naissensis. 2018;35:65-72. doi:10.2478/afmnai-2018-0007

\section{Publish your work in this journal}

Patient Preference and Adherence is an international, peer-reviewed, open access journal that focusing on the growing importance of patient preference and adherence throughout the therapeutic continuum. Patient satisfaction, acceptability, quality of life, compliance, persistence and their role in developing new therapeutic modalities and compounds to optimize clinical outcomes for existing disease states are major areas of interest for the journal. This journal has been accepted for indexing on PubMed Central. The manuscript management system is completely online and includes a very quick and fair peer-review system, which is all easy to use. Visit http://www.dovepress.com/testimonials.php to read real quotes from published authors.

Submit your manuscript here: https://www.dovepress.com/patient-preference-and-adherence-journal 